\title{
Three-Dimensional Biplanar Reconstruction of Scoliotic Rib Cage Using the Estimation of a Mixture of Probabilistic Prior Models
}

\author{
Said Benameur*, Max Mignotte, François Destrempes, and Jacques A. De Guise
}

\begin{abstract}
In this paper, we present an original method for the three-dimensional (3-D) reconstruction of the scoliotic rib cage from a planar and a conventional pair of calibrated radiographic images (postero-anterior with normal incidence and lateral). To this end, we first present a robust method for estimating the model parameters in a mixture of probabilistic principal component analyzers (PPCA). This method is based on the stochastic expectation maximization (SEM) algorithm. Parameters of this mixture model are used to constrain the 3-D biplanar reconstruction problem of scoliotic rib cage. More precisely, the proposed PPCA mixture model is exploited for dimensionality reduction and to obtain a set of probabilistic prior models associated with each detected class of pathological deformations observed on a representative training scoliotic rib cage population. By using an appropriate likelihood, for each considered class-conditional prior model, the proposed 3-D reconstruction is stated as an energy function minimization problem, which is solved with an exploration/selection algorithm. The optimal 3-D reconstruction then corresponds to the class of deformation and parameters leading to the minimal energy. This 3-D method of reconstruction has been successfully tested and validated on a database of 20 pairs of biplanar radiographic images of scoliotic patients, yielding very promising results. As an alternative to computed tomography-scan 3-D reconstruction this scheme has the advantage of low radiation for the patient, and may also be used for diagnosis and evaluation of deformity of a scoliotic rib cage. The proposed method remains sufficiently general to be applied to other reconstruction problems for which a database of objects to be reconstructed is available (with two or more radiographic views).
\end{abstract}

Index Terms-Biplanar radiographies, medical imaging, mixtures of probabilistic principal component analyzers, reduction of dimensionality, scoliosis, shape model, stochastic optimization, 3-D reconstruction model, 3-D/2-D registration.

Manuscript received March 26, 2004; revised January 23, 2005. This work was supported by the Natural Sciences and Engineering Research Council of Canada under Project 107998-99), in part by the Canadian Foundation for Innovation, Valorisation recherche Québec under Project 2200-003, and in part by the Biospace Company, Paris, France. Asterisk indicates corresponding author.

*S. Benameur is with the Laboratoire de recherche en imagerie et orthopédie, University of Montréal Hospital Research Centre, Montréal, QC H2L 2W5, Canada. He is also with the Laboratoire de vision et modélisation géométrique (DIRO), University of Montréal, Montréal, QC H2L 2W5, Canada (e-mail: benameus@iro.umontreal.ca).

M. Mignotte is with the Laboratoire de vision et modélisation géométrique (DIRO), University of Montréal, Montréal, QC H2L 2W5, Canada. He is also with the Laboratoire de recherche en imagerie et orthopédie (LIO), University of Montréal Hospital Research Centre, Montréal, QC H2L 2W5, Canada.

F. Destrempes is with the Laboratoire de vision et modélisation géométrique (DIRO), University of Montréal, Montréal, QC H2L 2W5, Canada.

J. A. De Guise is with the Laboratoire de recherche en imagerie et orthopédie (LIO), University of Montréal Hospital Research Centre, Montréal, QC H2L 2W5, Canada. He is also with the Automated Production Department of the École de technologie supérieure of Montréal, Montréal, QC H2T 2C8, Canada.

Digital Object Identifier 10.1109/TBME.2005.855717

\section{INTRODUCTION}

$\mathbf{S}$ COLIOSIS is a three-dimensional (3-D) deformity of the natural curve of the spinal column which can occur at any time during a child's growth. Due to its interaction with the spinal column, the abnormal curvature of the spine will cause a complex deformation of the rib cage including rotation and distortions. If this disease is not treated, a child's heart and lungs will not work properly as the twisted rib cage will not allow them enough space to develop. The rib cage plays also another important role in the protection of several other internal organs and of allowing motion of the trunk in respiration as well as spinal flexion.

In this paper, we are concerned with automatic 3-D reconstruction methods of scoliotic rib cage from a pair of planar and conventional calibrated radiographic images (postero-anterior with normal incidence and lateral). This 3-D geometrical information is useful for the diagnosis (i.e., to quantify a deformity of a scoliotic rib cage), surgical planing, computer assisted surgery or to quantify the postoperative 3-D changes of a scoliotic rib cage [1].

Recent imaging modalities and devices make it possible to obtain a 3-D reconstruction of surface shape from scanned anatomic structure, creating detailed and realistic 3-D surface representations of the scanned anatomic structure and allowing 3-D study of external pathologic deformities. These imaging devices used different 3-D reconstruction methods. Among these methods, the 3-D reconstruction methods of tomodensitometric imagery modalities (e.g., computerized tomography [2], [3], or magnetic resonance [4], [5]) provide accurate 3-D information of the human anatomy. However, few hospitals can afford a magnetic resonance system, it is time consuming, and the presence of metallic implants, once surgery has been performed, produces artifacts and creates suboptimal images. Tomodensitometric imagery systems can now be found in every hospital and many clinics. Nevertheless, the high level of X-ray dose required to provide full 3-D data or large bone structures as the rib cage make them less functional. Also, these imaging modalities and devices require that the patient be in a lying position, which is incompatible with many diagnostic protocols evaluating scoliosis. For these above-mentioned reasons, a 3-D reconstruction method of shapes using a limited number of X-ray radiographic projections and thus ensuring a lower amount of radiation for the patient, is really interesting.

The 3-D reconstruction problem of (scoliotic) rib cage from two or several projections has not been widely studied and few references exist in the literature. 


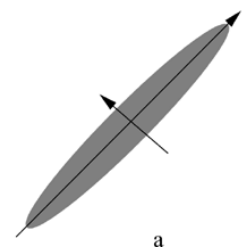

a

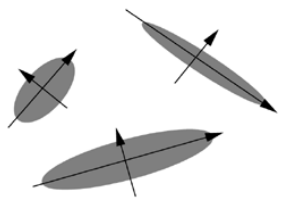

b
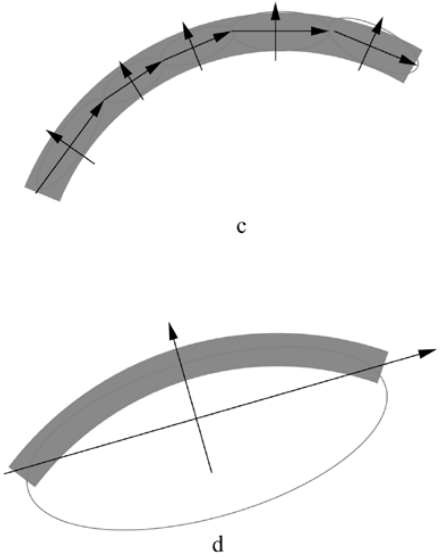

Fig. 1. An illustration of adjustment of a distribution. The grey area designates the distribution of the sample to be estimated and the ellipse represents the level set of a Gaussian distribution associated to each estimated PCA. (a) and (d) Adjustment with PCA (not optimal in this latter case), (b) and (c) adjustment with mixtures of PPCA.

One of the first 3-D reconstruction technique using two radiographic projections [namely, postero-anterior with normal incidence $\left(I_{\mathrm{PA}}\right)$ and postero-anterior with $20^{\circ}$ angled down incidence $\left(I_{\mathrm{PA}-20^{\circ}}\right)$ ] was proposed in [6]. This stereo-radiographic method consists in identifying 11 midline points per rib on $I_{\mathrm{PA}}$ and $I_{\mathrm{PA}-20^{\circ}}$. 60 points are interpolated on the two-dimensional (2-D) curve of the rib on $I_{\mathrm{PA}}$. 3-D points are obtained by intersection of a projection plane, which is defined using the interpolated points and the $3-\mathrm{D}$ position of $I_{\mathrm{PA}}$ and $I_{\mathrm{PA}-20^{\circ}} \mathrm{X}$-ray sources, and the 2-D curve of the same rib on $I_{\mathrm{PA}-20^{\circ}}$ using a direct linear transformation (DLT) [7]. This process is repeated for each of the 60 points of all rib defined on $I_{\mathrm{PA}}$. This method is then refined by taking into account patient displacement during stereo-radiography [8]. Nevertheless, this technique remains limited due to the inherent inaccuracy produced in identifying 11 points per rib on $I_{\mathrm{PA}}$ and $I_{\mathrm{PA}-20^{\circ}}$ (leading to reconstruction errors). Locating these features is widely supervised and, therefore, time consuming (up to two hours). Moreover, this method does not exploit all information contained in the two X-ray radiographic projections, for instance, the contours of each rib, the geometrical structure or the statistical knowledge of the possible deformation of the rib cage to be reconstructed.

Three-dimensional reconstruction methods using a priori knowledge of the geometric shape of the objects of interest have been recently proposed. For example, a method similar to the one reported by Benameur et al. in [9] for the reconstruction of each vertebra of the spinal column was proposed by Mouren [10] to reconstruct the 3-D geometry of the rib cage from two radiographic projections [postero-anterior with normal incidence $I_{\mathrm{PA}}$ and lateral $\left.\left(I_{\mathrm{LAT}}\right)\right]$. This method exploits a global prior knowledge on the geometrical structure of each rib. To find this geometrical knowledge while reducing the dimensionality of this problem, a principal component analysis (PCA) is applied to each rib extracted from a training scoliotic rib cage database. This PCA allows to obtain a geometric prior model representing a mean shape and containing the typical geometrical deformation modes estimated in the least square sense. Thereafter, the rib model is deformed according to the rays back projected from the contour points of the projections taken a patient. In $I_{\mathrm{LAT}}$, a set of 60 points are manually digitized. This 3 -D reconstruction is done rib by rib. This method is interesting but may not be very accurate. First, it is significantly supervised and operator-dependent; it requires to identify manually and digitize a set of 60 points in the lateral view. Moreover, the PCA only defines a linear dimensionality reduction which is a strong and not necessarily a true assumption in this context (e.g., Fig. 1). In order to overcome the problem of supervision and improve the accuracy of the deformation model, we propose herein to use a mixture of Probabilistic PCA [11] (PPCA). In order to estimate the parameters of such a mixture model, the expectation-maximization (EM) algorithm was already proposed [11]. Nevertheless, the initial parameter values have a significant impact on the convergence of this iterative procedure and on the quality of the final estimation (the EM converges to a local and not necessary global optimum estimate [12]). In order to make the procedure more robust, we propose a stochastic version of the EM-PPCA relying on the Stochastic version of the EM (SEM) algorithm [13], [14]. The SEM algorithm has been shown to be computationally less burdensome and more appropriate than the EM algorithm for the problems of mixture estimation and missing data [12], [13], [15], [16]. This SEM-PPCA is efficiently exploited for dimensionality reduction and to get a set of probabilistic prior models, associated to each detected class of pathological deformations, observed on a representative training scoliotic rib cage population. For each considered class-conditional prior model of pathological deformations, the proposed reconstruction method then consists in fitting the projections of an instance of the deformation model with the segmented contours of the corresponding rib cage on the two radiographic views. This matching problem leads to a set of $K$ optimization problems (one for each detected class of pathological deformations) which is efficiently solved in our application with a exploration/selection algorithm. The optimal 3-D reconstruction corresponds to the solution, leading to the minimal energy, amongst these $K$ optimization problems.

MPPCA models remains a standard Finite Normal Mixture (FNM) problem, which has been exhaustively studied for several decades and applied to many fields. Parameter estimation of FNM via EM algorithm or its variants has also been studied thoroughly. Lei and Sewchand in [17] has developed a hybrid algorithm for FNM parameter estimation which uses classification maximization (CM) algorithm (a modified $K$-means clustering procedure [17]) to generate the initial parameter estimates and EM algorithm to produce the final estimates. Lei and Udupa in [18] also shows that $K$-means clustering procedure is a special case of EM algorithm for FNM parameter estimation.

Let us also note that probabilistic PCA (PPCA) has been already used in shape localization in images [19], object classification [20] and inference of 3-D structure [21]. Nevertheless, mixture of PPCA has only been exploited in image compression [11]. To our knowledge, this work is the first to use mixtures of probabilistic PCA for the 3-D modeling of a class of complex shapes and to constrain the reconstruction problem in medical imagery.

The remainder of this paper is organized as follows. In Section II, we present the basic concept of a mixture of probabilistic PCA. Section III is devoted to the stochastic EM algorithm for estimating all of the model parameters in a mixture of probabilistic PCA. Sections IV and V describe the 
proposed 3-D reconstruction method and the related sequence of energy function to be minimized. Section VI presents the validation protocol of our method. In Section VII, we show some 3-D reconstruction results and validate the proposed model.

\section{Probabilistic Model For Dimensionality Reduction}

PCA is a well-established model for dimensionality reduction. Nevertheless, one limiting disadvantage of this technique is the absence of an associated probability density model or generative model [11]. PPCA overcomes this problem.

\section{A. Probabilistic PCA}

Tipping and Bishop have developed the PPCA model [11], [22] by reformulating PCA as a maximum-likelihood (ML) solution of a specific form of variable model. Let $s$ and $b$ be two random vectors related by

$$
s=\Phi b+\nu+\varepsilon
$$

where $\Phi$ is a $d \times m$ matrix that represents the principal subspace of $s$. The assumption is that $b \sim \mathcal{N}\left(0, I_{m}\right)$ as well as $\varepsilon \sim$ $\mathcal{N}\left(0, \sigma^{2} I_{d}\right)$ are zero mean Gaussian distributed random vectors ( $I_{r}$ denotes the $r \times r$ identity matrix and $\mathcal{N}()$ represents the normal distribution). Consequently, the random vector $s$ is also normally distributed according to

$$
p_{S}(s)=\mathcal{N}\left(s ; \nu, \sigma^{2} I_{d}+\Phi \Phi^{T}\right) .
$$

The variable $s$ represents the full data, whereas $b$ represents the reduced data (the dimension $m$ of $b$ is significantly smaller than the dimension $d$ of $s$ ).

Let $\left[s_{1}, s_{2}, \ldots, s_{u}\right]^{T}$ be a independent identically distributed (i.i.d.) observation sample issued from $s, \lambda_{1}, \ldots, \lambda_{d}$ be the eigenvalues of the covariance matrix, in decreasing order, of this sample, $\Lambda_{m}$ be the diagonal matrix with entries $\lambda_{1}, \ldots, \lambda_{m}$, and $U_{m}$ be the $d \times m$ matrix with columns equal to the corresponding eigenvectors, normalized so that they have euclidean norm equal to 1 . From [11], a ML estimation of $\left(\nu, \sigma^{2}, \Phi\right)$ is given by

$$
\begin{aligned}
\nu & =\bar{s}=\frac{1}{u} \sum_{i=1}^{u} s_{i} \\
\sigma^{2} & =\frac{1}{d-m} \sum_{i=m+1}^{d} \lambda_{i} \\
\Phi & =U_{m}\left(\Lambda_{m}-\sigma^{2} I_{m}\right)^{1 / 2} .
\end{aligned}
$$

Note that in the PCA, one would take $\Phi=U_{m}$, but this choice is not optimal in the sense of the ML for the PPCA model. The diagonal matrix $\left(\Lambda_{m}-\sigma^{2} I_{m}\right)^{1 / 2}$ gives an appropriate weight to each column vector of the matrix $U_{m}$.

The conditional probability distribution of $s$ given $b$, is expressed by [11]

$$
P_{S / B}(s / b)=\left(2 \pi \sigma^{2}\right)^{-1 / 2} \exp \left(-\frac{1}{2 \sigma^{2}}\|s-W-\bar{s}\|\right)
$$

where $W=U_{m}\left(\Lambda_{m}-\sigma^{2} I_{m}\right)^{1 / 2} b$. Hence, the ML reconstructed data point is taken as

$$
\hat{s}=U_{m}\left(\Lambda_{m}-\sigma^{2} I_{m}\right)^{1 / 2} b+\bar{s} .
$$

In that case, the reduction map is defined by

$$
\hat{b}=\left(\Lambda_{m}-\sigma^{2} I_{m}\right)^{-1 / 2} U_{m}^{t}(s-\bar{s})
$$

in order to minimize the average reconstruction error (optimal in the least square sense)

$$
e=\frac{1}{n} \sum_{i=1}^{n}\left\|s_{i}-U_{m} U_{m}^{t}\left(s_{i}-\bar{s}\right)-\bar{s}\right\|^{2} .
$$

These reconstruction and reduction maps were adopted in [23]. We summarize the PPCA algorithm in Algorithm 1.

\section{B. Mixtures of Probabilistic Principal Component Analysis}

The probabilistic formulation of PCA offers a graceful extension to model complex data structures with a mixture of local PPCA models. A mixture of PPCA (MPPCA) is a distribution of the form [11]

$$
P_{S}(s)=\sum_{c=1}^{K} \pi_{c} \mathcal{N}\left(s ; \nu_{c}, \sigma_{c}^{2} I_{d}+\Phi_{c} \Phi_{c}^{T}\right)
$$

where $K$ designates the number of components of the mixture and $\pi_{c}$ is the $c$ th component's mixing proportion. This mixture depends on a vector of parameters $\Gamma=\left(\nu_{1}, \sigma_{1}, \Phi_{1}, \ldots, \nu_{K}, \sigma_{K}, \Phi_{K}\right)$. In order to reduce dimensionality, the following strategies can be adopted; we compute for each mixture component $c$, the reconstruction point $\overline{s_{c}}$ and take the one nearest to $s$ as the one that maximizes the distribution $P_{S}(s)$ (optimality in the ML sense).

\section{ESTIMATION OF A MiXTURE OF PPCA}

In order to estimate parameter vector $\Gamma$, we resort to the SEM algorithm [13]. The SEM algorithm is an iterative algorithm where at each iteration the parameters of the mixture are estimated in the ML sense. This iterative procedure requires initial parameters which can be given by a $K$-means clustering procedure [24] (in doing so, we assume as first approximation that the considered clusters are spherical with equal volumes). The obtained spherical partitions allow to obtain a rough estimation of the mixture parameters which are then used to initialize the SEM clustering and estimation procedure. See Algorithm 1, PPCA algorithm, at the top of the next page.

\section{A. K-Means Algorithm}

The $K$-means algorithm consists of the following steps. To initialize $K$ clusters, choose $K$ shapes at random from the training base. For each shape selected in the training base, assign this shape to a class such that the Euclidean distance from this shape to the center of that cluster is minimal. For each cluster, recalculate the means of the cluster based on the shapes that belong to that cluster. This second step is running until the center of each class becomes steady.

\section{B. Stochastic EM Algorithm}

Let $K$ be an upper bound on the number of classes and $\xi \epsilon$ ]0, 1 [ be a chosen threshold. In the context of the estimation of a MPPCA, the SEM algorithm can be outlined as follows, (the superscript denoting the iteration number).

\section{Initialization}

We initialize the SEM algorithm by the ML parameters estimated on the full data obtained by a $K$-means [24] partition 


\section{PPCA Algorithm}

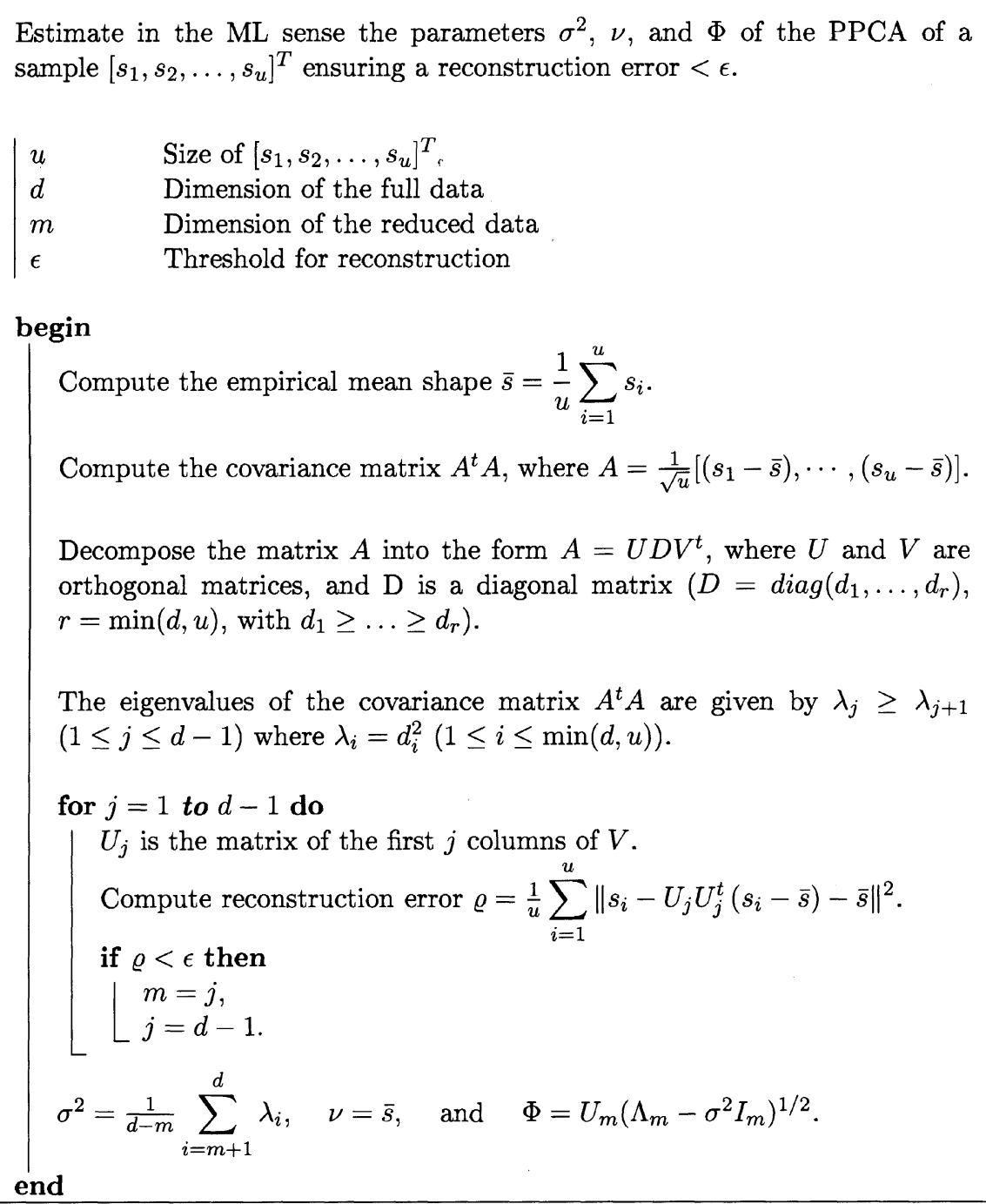

Algorithm 1: PPCA algorithm.

of $\left[s_{1}, s_{2}, \ldots, s_{u}\right]^{T}$ into $K$ classes. For every observation $s_{i}$, the probability $P_{S / C}^{[0]}\left(s_{i} / c\right)$ of its belonging to the class $c,(c \in$ $\{1, \ldots, K\})$ can then be defined. This terminates the initialization phase.

An iteration of SEM then consists of three steps.

Step $\mathbf{S}$ (Stochastic)

For each $s_{i}$, we sample from the set of classes an element according to the distributions $\left[P_{C / S}^{[p]}\left(1 / s_{i}, \Gamma^{[p]}\right), \ldots, P_{C / S}^{[p]}\left(K / s_{i}, \Gamma^{[p]}\right)\right]$.

This selection defines a partition $\left[\vartheta_{1}^{[p]}, \ldots, \vartheta_{K}^{[p]}\right]$ of the sample $\left[s_{1}, s_{2}, \ldots, s_{u}\right]^{T}$.

Step M (Maximization)

The SEM algorithm supposes that every $s_{i}$ belonging to $\vartheta_{c}^{[p]}$ for each $c \in[1, K]$, is realized according to the distribution defined by $P_{S / C}\left(s_{i} / c, \Gamma\right)$ [see (1)], the density corresponding to class $c$. Let $u$ be the number of rib cages in the training base and $\hat{\pi}_{c}^{[p+1]}$ be the $c$ th component's mixing proportion at iteration $[p+1]$. The parameters of the mixture are estimated for the full data in the ML sense upon setting $\hat{\pi}_{c}^{[p+1]}=\operatorname{Card}\left(\vartheta_{c}^{[p]}\right) / u$ $\left(\operatorname{Card}\left(\vartheta_{c}^{[p]}\right)\right.$ stands for the cardinal or the number of component of $\vartheta_{c}^{[p]}$ ) and estimating $\Gamma^{[p+1]}$ on each class according to [see (2)]. If $\hat{\pi}_{c}^{[p+1]}<\xi$, we eliminate the class $c$ in the mixture. ${ }^{1}$

Step E (Estimation)

For each $s_{i}$, we define the next distribution $\left[P_{C / S}\left(1 / s_{i}, \Gamma^{[p+1]}\right), \ldots, P_{C / S}\left(K / s_{i}, \Gamma^{[p+1]}\right)\right] \quad$ by the posterior distribution based on the current parameter $\Gamma^{[p+1]}$

$$
P_{C / S}\left(c / s_{i}, \Gamma^{[p+1]}\right)=\frac{\pi_{c}^{[p+1]} P_{S / C}\left(s_{i} / c, \Gamma^{[p+1]}\right)}{\sum_{c=1}^{K} \pi_{c}^{[p+1]} P_{S / C}\left(s_{i} / c, \Gamma^{[p+1]}\right)} .
$$

Return to Step S until a fixed maximum number of iterations has been reached.

${ }^{1}$ There are many other unsupervised ways to estimate the number of classes $K$, e.g., by using information theoretic criteria [Akaike information criterion (AIC) or maximum description lenght (MDL)] [17], [18], [25], [26]. 


\section{Time Complexity of Stochastic EM Algorithm}

The time complexity of $K$-means is proportional to the number $K$ of clusters to be found. More precisely, the $K$-means time complexity is $O(n K u d)$ where $u$ is the number of rib cages, $d$ is the dimensionality of each rib cage, and $n$ is the number of iterations required. The time complexity of the $K$-means empirically grows linearly with $K, u$, and $d$ [27]. Consequently, the time complexity of the initialization step of SEM is $O(n K u d)$.

The stochastic step of the SEM procedure is in fact similar to the Gibbs sampling step [28]. The time complexity of stochastic step of SEM is $O\left(u d^{2}\right)$ for one cluster (or one class).

The maximization step consists in computing the ML estimates of parameters associated to each cluster, using PPCA algorithm, based on the data currently classified in the corresponding cluster. It is thus equivalent to estimate the time complexity of $K$ PPCA estimations. To this end, we use a singular value decomposition (SVD) which can be done by using the Golub-Reinsch algorithm with time complexity $O\left(u^{2} d+u d^{2}+\right.$ $d^{3}$ ) [29]. The parameters of the mixture are then estimated in the ML sense with time complexity equal to $O\left(d^{2}+d^{3}\right)$ for each cluster.

The time complexity of the SEM-PPCA algorithm is thus $N K O\left(u^{2} d+u d^{2}+d^{3}\right)$ for $N$ iterations of the SEM procedures and $K$ clusters.

\section{MiXture of Statistical Deformable Models}

The shape $s$ of each rib cage of our training database described in Section VII-A is defined by a set of $l$ control points, or landmarks, ${ }^{2}$ which approximate the geometrical shape of the midline of each rib in $\mathcal{R}^{3}$. Each rib cage in the training set is thus represented by the following $3 l$-dimensional vector

$$
s=\left(x_{1}, y_{1}, z_{1}, \ldots, x_{l}, y_{l}, z_{l}\right)^{t}
$$

where $\left(x_{i}, y_{i}, z_{i}\right)^{t}$ are the Cartesian coordinates of the $i$ th control point or landmark of the shape.

\section{A. Training Phase}

First, we extend into 3-D the method proposed by Cootes in [30] for aligning the set of $u 3$-D shape samples $\left[s_{1}, s_{2}, \ldots, s_{u}\right.$ ] of our training database to a common scale, rotation, and translation. This step is important and allows to eliminate the variations introduced by affine deformations such as translation, scaling, and rotation.

To this end, an arbitrary shape is selected as the initial average shape estimate. All the other shapes are aligned to this average using a least square minimization. A new average is computed by a simple mean across the corresponding points, and the procedure repeats until convergence.

\footnotetext{
${ }^{2}$ These landmarks are obtained with the following procedure: from a pair of stereo-radiographic images (namely, postero-anterior with normal incidence $\left(I_{\mathrm{PA}}\right)$ and postero-anterior with $20^{\circ}$ angled down incidence $\left(I_{\mathrm{PA}-20^{\circ}}\right)$ ) we manually identify 11 midline points per rib on $I_{\mathrm{PA}}$ and $I_{\mathrm{PA}-20^{\circ}} .60$ points are interpolated on the 2-D curve of the rib on $I_{\mathrm{PA}}$ [6]. 3-D points are obtained by intersection of a projection plane, which is defined using the interpolated points and the 3-D position of $I_{\mathrm{PA}}$ and $I_{\mathrm{PA}-20^{\circ}} \mathrm{X}$-ray sources, and the 2-D curve of the same rib on $I_{\mathrm{PA}-20^{\circ}}$ using a Direct Linear Transformation (DLT) [7]. This process is repeated for each of the 60 points of all rib defined on $I_{\mathrm{PA}}$.
}

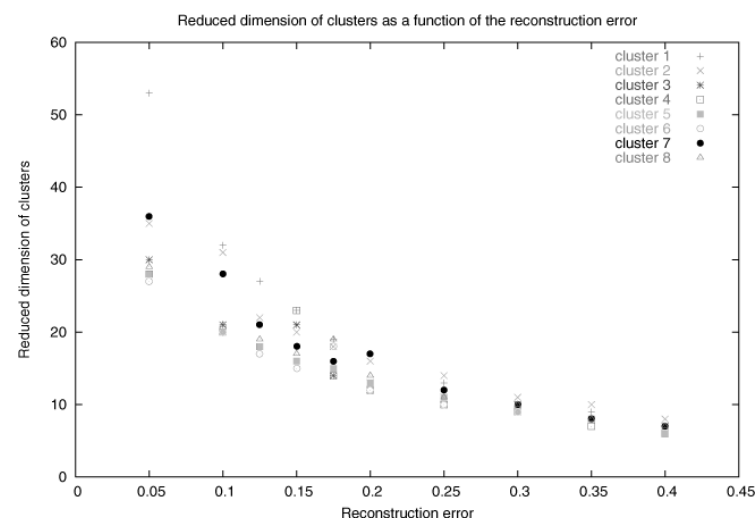

(a)

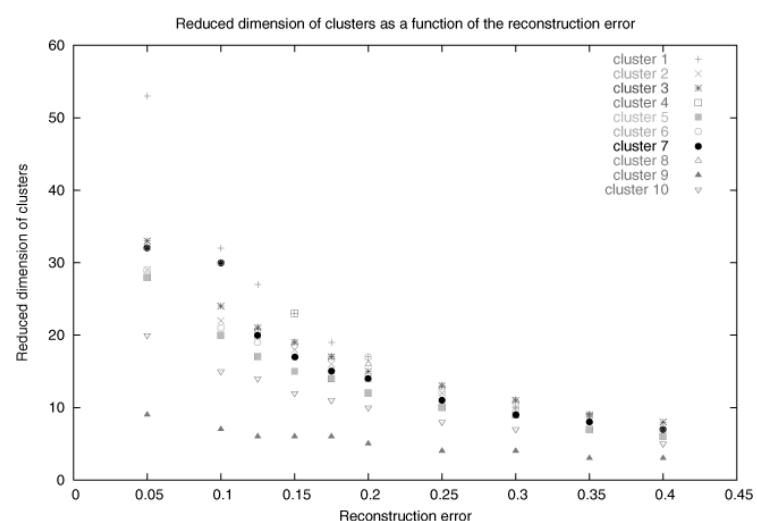

(b)

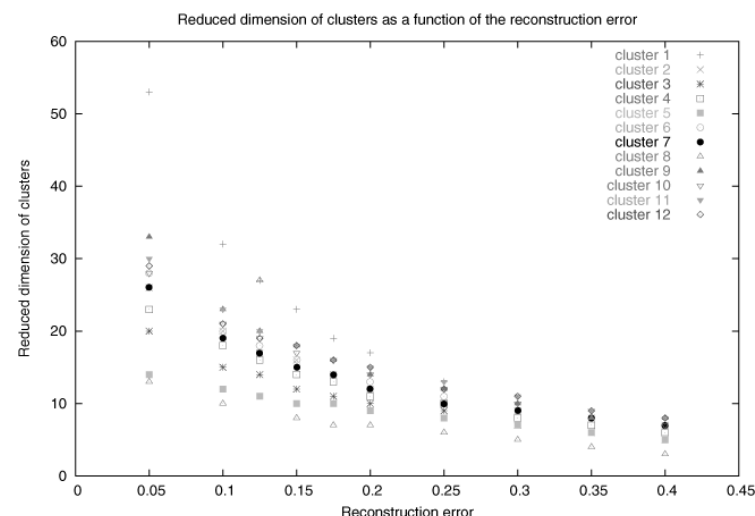

(c)

Fig. 2. Reduced dimension of clusters as a function of the reconstruction error using different number of clusters the rib cage database is partitioned into (a) 8 , (b) 10 , and (c) 12 clusters.

Any scaling and rotation in 3-D can be expressed as quaternion $^{3}$ where scaling is expressed by the magnitude of the quaternion and the 3-D rotation is expressed by the direction of the unit vector $u$ and rotation $\varphi$. Quaternion have some

${ }^{3} \mathrm{~A}$ quaternion $q$ is defined as the linear combination of a scalar term $q_{0} \geq 0$ and three right-handed orthonormal vectors $(i, j$, and $k$ ) $q=q_{0}+q_{1} i+q_{2} j+q_{3} k$. The magnitude of the quaternion is defined as, $|q|=\sqrt{q_{0}^{2}+q_{1}^{2}+q_{2}^{2}+q_{3}^{2}}$, and any unit length quaternion can be written as, $q=\cos (\varphi) \cdot u+\sin (\varphi) \cdot u$, where $u$ is a unit vector and $\varphi$ represents a rotational twist along the unit vector. The Cartesian rotation matrix is given by [31]

$$
R=\left(\begin{array}{ccc}
q_{0}^{2}+q_{1}^{2}-q_{2}^{2}-q_{3}^{2} & 2\left(q_{1} q_{2}-q_{0} q_{3}\right) & 2\left(q_{1} q_{3}+q_{0} q_{2}\right) \\
2\left(q_{1} q_{2}+q_{0} q_{3}\right) & q_{0}^{2}+q_{2}^{2}-q_{1}^{2}-q_{3}^{2} & 2\left(q_{2} q_{3}-q_{0} q_{1}\right) \\
2\left(q_{1} q_{3}-q_{0} q_{2}\right) & 2\left(q_{2} q_{3}+q_{0} q_{1}\right) & q_{0}^{2}+q_{3}^{2}-q_{1}^{2}-q_{2}^{2}
\end{array}\right)
$$




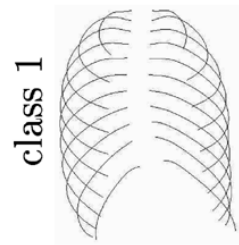

\section{Mean Shape}
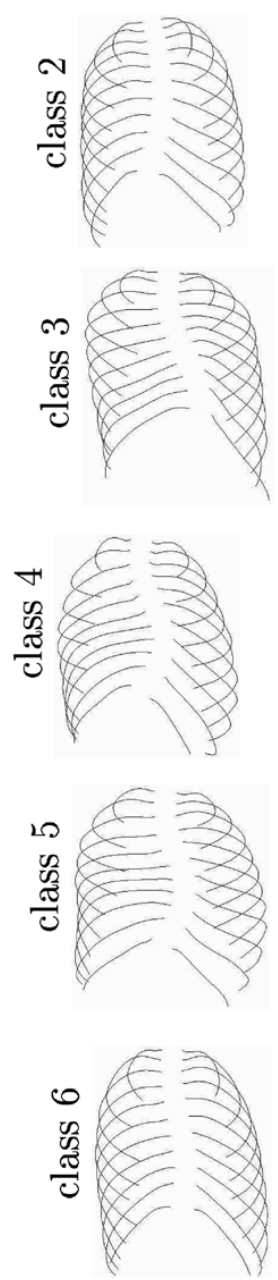

$-1$
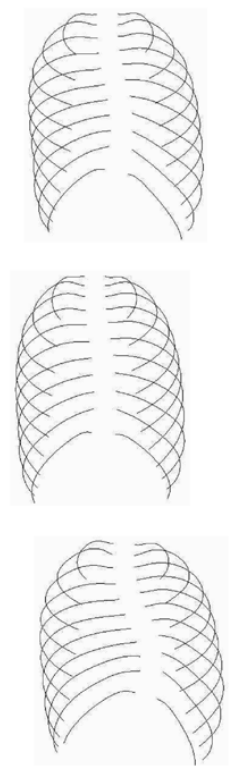
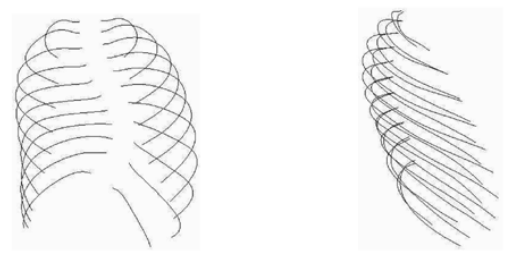

Mean Shape
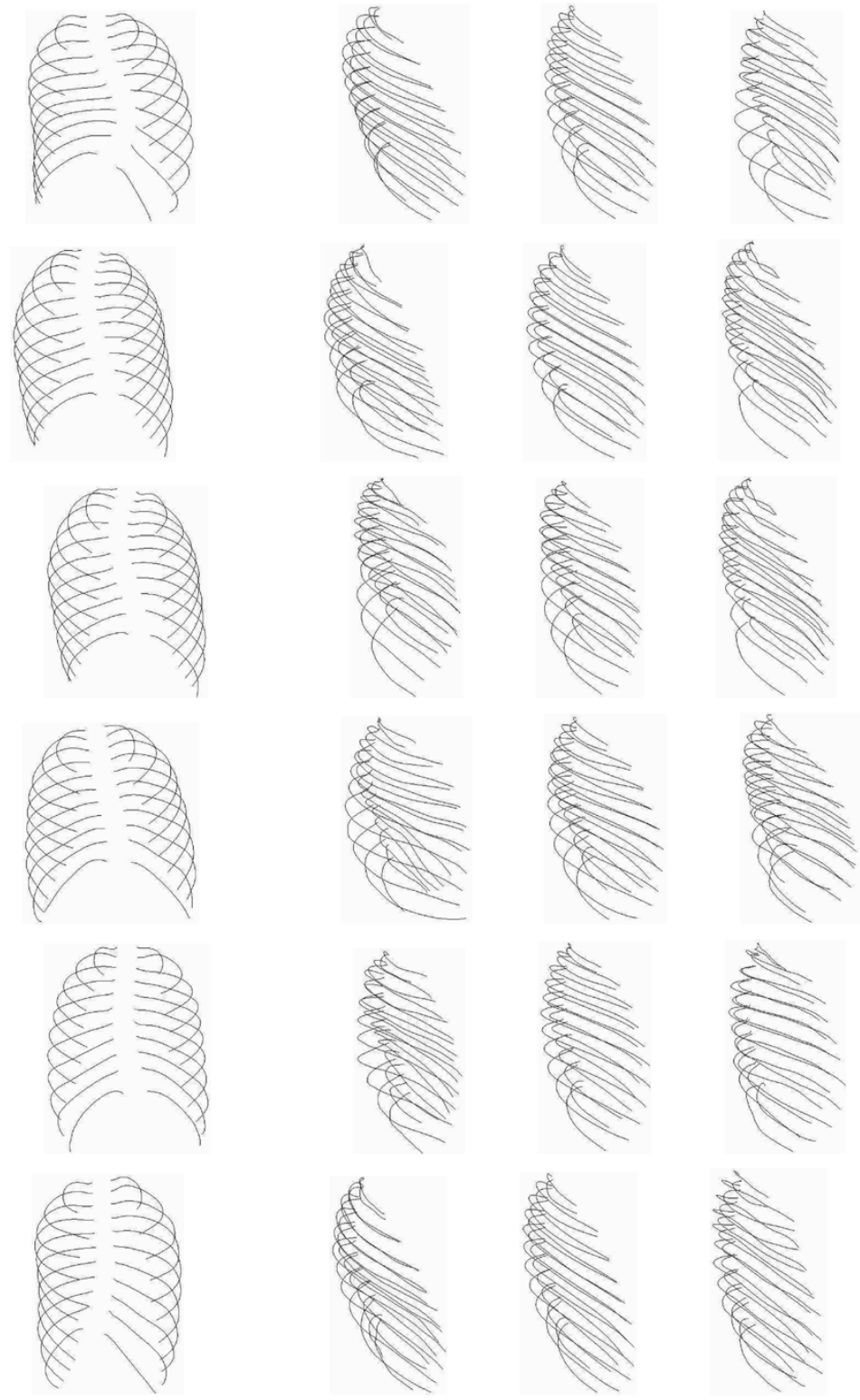

1

$-1$

1

Fig. 3. Prior model of each class. Two deformed shapes obtained by applying \pm 1 standard deviation of the first deformation mode associated to each class to the mean shape (sagital and coronal views).

advantages amongst which simple derivatives and efficient formulations to find rotation matrices directly for point matching problems [32]. The alignment of two 3-D shape instances is accomplished using a well-known procedure given in [32] to optimize for $q$ and $t$.

We then apply the MPPCA to estimate the parameters of each class and to reduce the dimension to $m_{c} \ll 3 l$ (e.g., Fig. 2). Viewing $s_{c}=\left(x_{1}, y_{1}, z_{1}, \ldots, x_{l}, y_{l}, z_{l}\right)$ as a random vector, we obtain, for each class $c(c \in[1, K])$, an optimal probabilistic deformable model in the ML sense of the form $S_{c}=\Phi_{c} b_{c}+$ $\nu_{c}+\varepsilon_{c}$ with $\varepsilon_{c} \sim \mathcal{N}\left(0, \sigma_{c}^{2} I_{l}\right), \Phi_{c}$ a $3 l \times m_{c}$ matrix, and $b_{c} \sim$ $\mathcal{N}\left(0, I_{m_{c}}\right)$, by taking $\nu_{c}=\bar{s}_{c}, \sigma_{c}^{2}=\left(\frac{1}{1-m_{c}}\right) \sum_{i=m_{c}+1}^{l} \lambda_{i}$, and $\Phi_{c}=U_{m_{c}}\left(\Lambda_{m_{c}}-\sigma_{c}^{2} I_{m_{c}}\right)^{1 / 2}$. This gives us nonlinear deformations of the mean shape of each pathological deformations detected in our representative learning set, and terminates the training phase (e.g., Figs. 3 and 4).

In our framework, we use the estimation of the MPPCA with $K$ classes as a preliminary classification step (and not as a compound density estimate) which is exploited in the reconstruction step.

\section{B. Deformation Parameters}

From the training phase, we obtain for each class $c(c \in[1, K])$, the mean shape $\bar{s}_{c}$, and its nonlinear deformations $s_{c}=\Phi_{c} b_{c}+\overline{s_{c}}$, where $\Phi_{c}=U_{m_{c}}\left(\Lambda_{m_{c}}-\sigma_{c}^{2} I_{m_{c}}\right)^{1 / 2}$ is the matrix of the first $m_{c}$ eigenvectors associated with the $m_{c}$ largest eigenvalues and $\mathbf{b}_{\mathbf{c}}=\left(b_{c, 1}, \ldots, b_{c, m_{c}}\right)^{t}$ is a vector containing the weights for these $m_{c}$ deformation modes. 


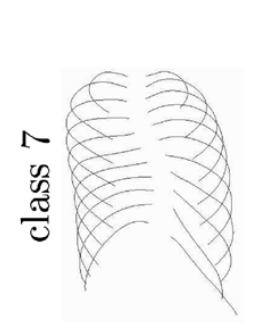

\section{Mean Shape}
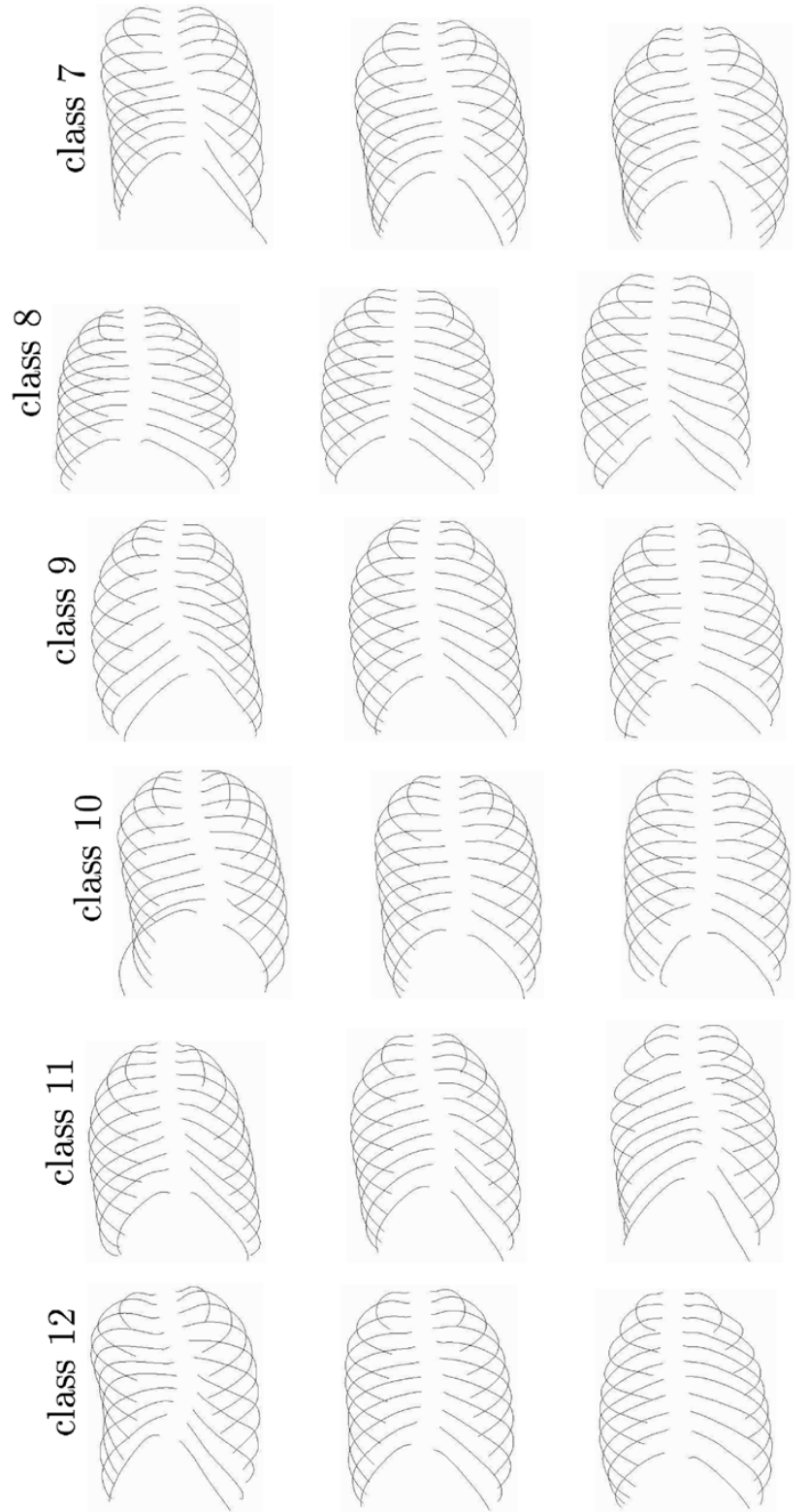

$-1$

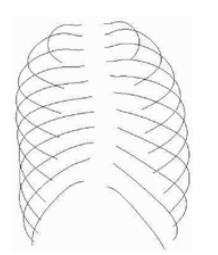

0
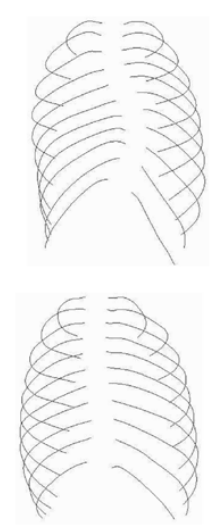

1
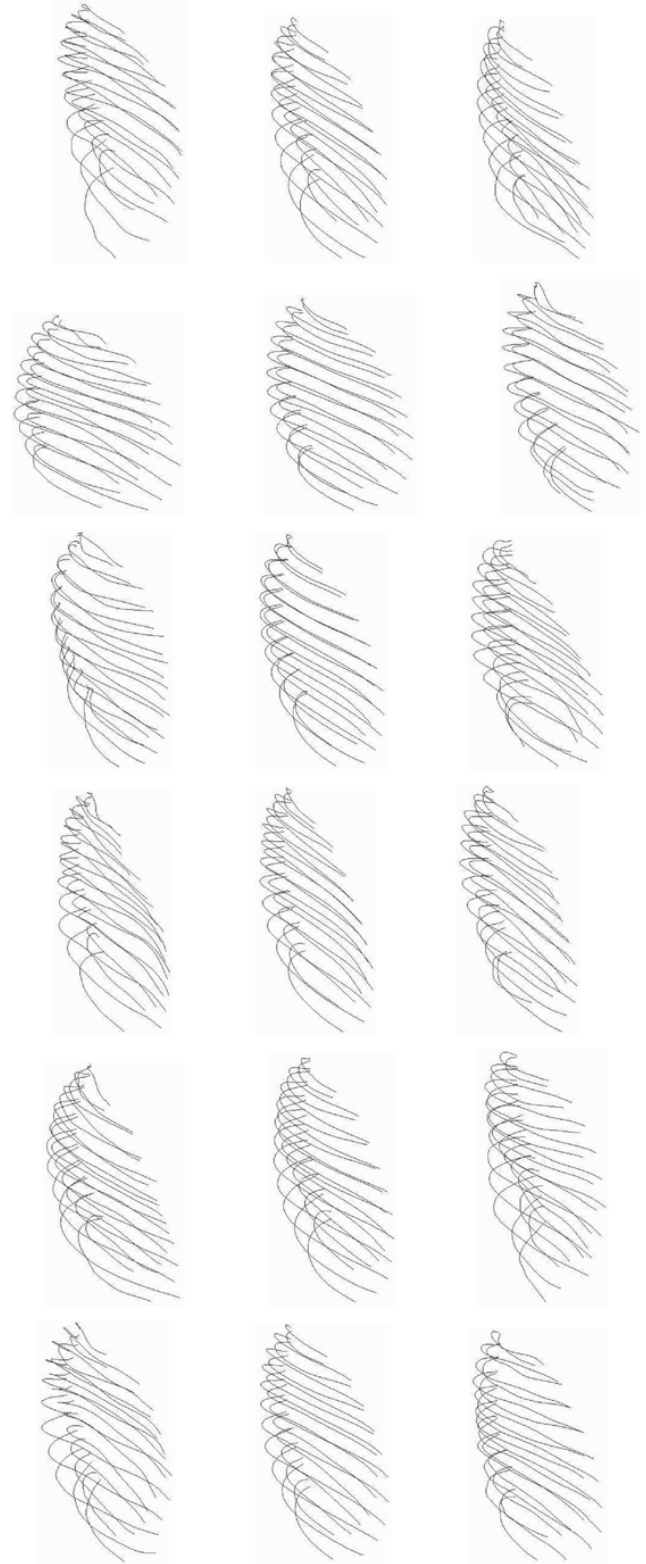

$-1$

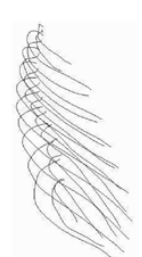

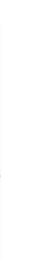
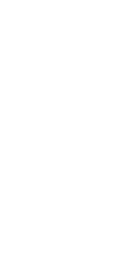

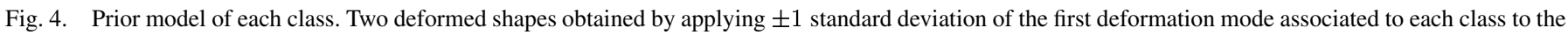
mean shape (sagital and coronal views).

In addition, we consider rigid deformations of the template given by global translation $T=\left(t_{x}, t_{y}, t_{z}\right)$, scaling $k=\left(k_{x}, k_{y}, k_{z}\right)$, and rotation $\alpha=\left(\alpha_{x}, \alpha_{y}, \alpha_{z}\right)$ around the $x$, $y$ and $z$, axis (applied point-wise to the template). This yields a vector of deformation

$$
\theta=\left(T, k, \alpha, b_{c}\right)
$$

of dimension $9+m_{c}$. The globally deformed template for class $c$ is then defined by

$$
s_{c}=M(k, \boldsymbol{\alpha})\left[\Phi_{c} b_{c}+\bar{s}_{c}\right]+T
$$

where $M(k, \alpha)$ performs a rotation and a scaling by $k$.

\section{Prior Energy Term}

Let $\Theta_{c}$ be the random variable corresponding to the vector of $\left(9+m_{c}\right)$ deformations parameters. We model the distribution of $\Theta_{c}$ by [19]

$$
P_{\Theta_{c}}\left(s_{c}(\theta)\right)=\mathcal{U}(T, k, \alpha) \exp \left(-\frac{1}{2} b^{t}\left(I_{m_{c}}-\sigma_{c}^{2} \Lambda_{m_{c}}^{-1}\right) b\right)
$$

where $\mathcal{U}$ is the uniform distribution with appropriate bounds for the 9 affine transformation parameters. This probability expresses the fact that the shape to be reconstructed is likely close to the mean shape. By considering that

$$
P_{\Theta_{c}}\left(s_{c}(\theta)\right)=\frac{1}{\zeta_{p}} \exp \left(-E_{p}\left(s_{c}(\theta)\right)\right)
$$




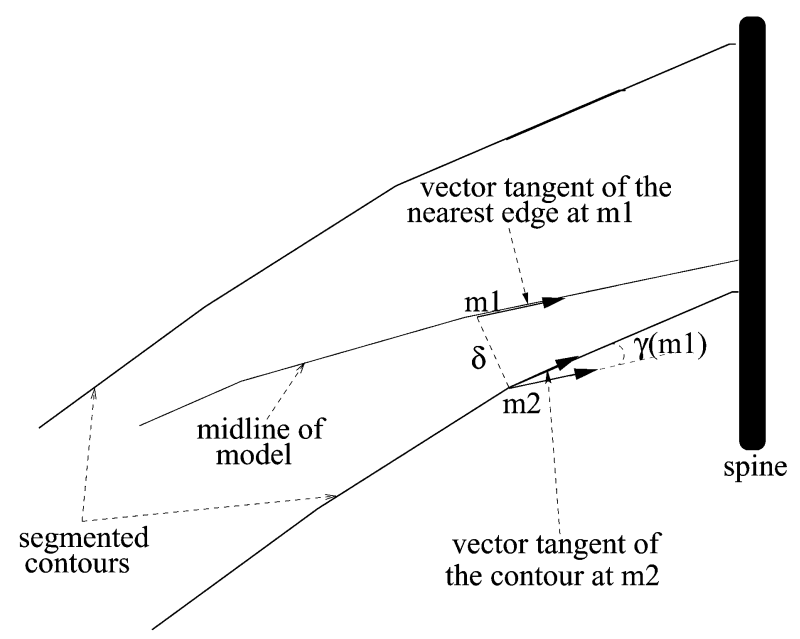

Fig. 5. Directional component used in the directional edge potential field $\Psi(x, y)$.

where $\zeta_{p}$ is a normalization constant, a prior energy term can be deduced from this low parametric representation

$$
E_{p}\left(s_{c}(\theta)\right)=\frac{1}{2} b^{t}\left(I_{m_{c}}-\sigma_{c}^{2} \Lambda_{m_{c}}^{-1}\right) b
$$

This energy term, which does not penalize affine transformations, will be used in our energy-based model to rightly constrain our 3-D reconstruction model by penalizing the deviation of the deformed template from the mean shape.

\section{Log-Likelihood Energy Term}

In a commonly used energy-based (deformable) model of reconstruction or segmentation, spatial contours (or some spatial gradient measures derived from the input image) are generally exploited in the data log-likelihood energy term. This term aims at expressing a measure of similarity between the deformed template and the object present in the image by constraining the deformable template to be attracted and aligned to the salient edges of the object to be detected [9]. Due to the low signal-to-noise ratio, low resolution and contrast of the radiographic images used in our application, the sharpness of the contour of each rib is noticeably reduced and our log-likelihood model cannot rely efficiently solely on this type of gradient measures. Instead, we propose a log-likelihood energy term exploiting an edge potential field [9] along with a statistical region-based homogeneity criterion. This measure is based on the computation of the grey-level variance inside and outside each rib.

To compute the edge potential field $\Psi_{\mathrm{PA}}$ associated with postero-anterior radiographic projection, we first use a Canny edge detector with the unsupervised technique proposed in [33]. Then, $\Psi_{\mathrm{PA}}$ is defined as in [34] by

$$
\Psi_{\mathrm{PA}}(x, y)=\exp \left(-\frac{\sqrt{\xi_{x}^{2}+\xi_{y}^{2}}}{\tau}\right)|\cos (\gamma(x, y))|
$$

where $\xi=\left(\xi_{x}, \xi_{y}\right)$ is the displacement to the nearest edge point in the image, and $\tau$ is a smoothing factor which controls the degree of smoothness of this potential field. $\gamma(x, y)$ is the angle between the tangent of the nearest edge and the tangent direction of the contour at $(x, y)$ (e.g., Fig. 5). To compute the statistical region-based homogeneity criterion $\Upsilon_{i}$ asso-

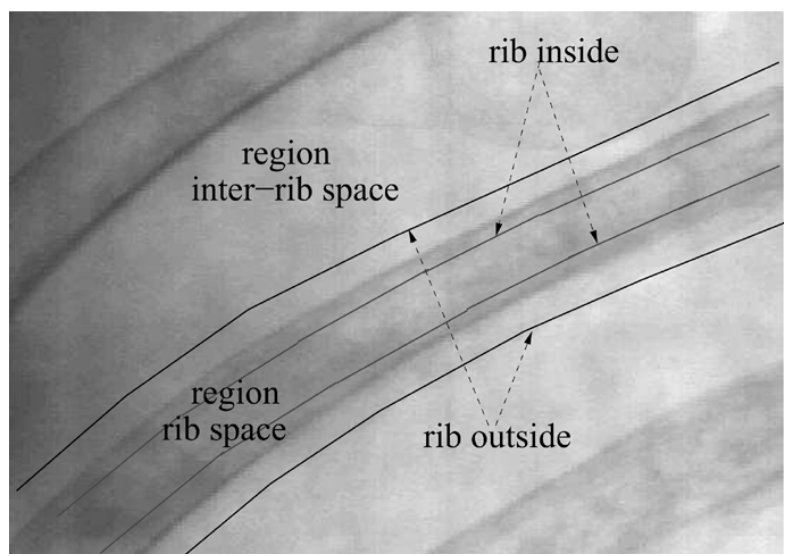

Fig. 6. An example demonstrating the use of statistical homogeneity criterion.

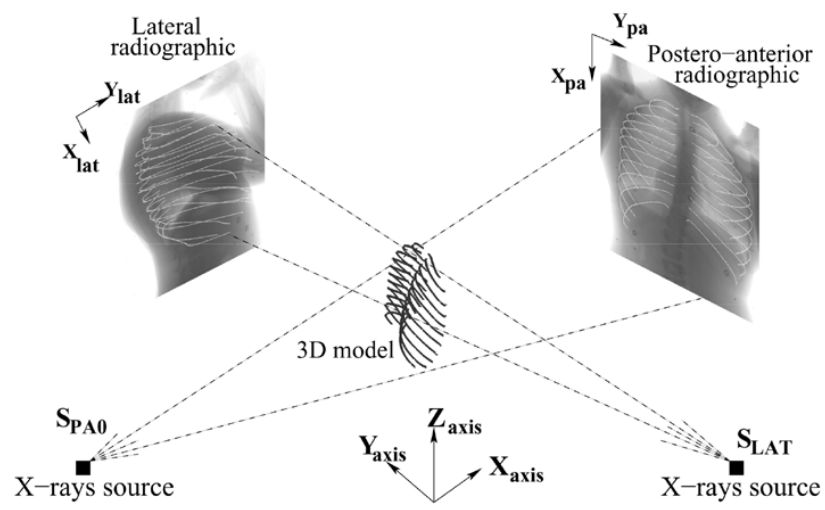

Fig. 7. Schematic example of a 3-D reconstruction of the scoliotic rib cage.

ciated with postero-anterior radiographic projection, we define the grey-level variance inside and outside for a rib $i$ by

$$
\Upsilon_{i}=\operatorname{var}_{\mathrm{Rib}_{i}^{\text {in }}}\left(I_{\mathrm{PA}}(x, y)\right)+\operatorname{var}_{\mathrm{Rib}_{i}^{\text {out }}}\left(I_{\mathrm{PA}}(x, y)\right)
$$

where $\operatorname{var}_{\mathrm{Rib}_{i}^{\text {in }}}$ (respectively, $\left.\operatorname{var}_{\mathrm{Rib}_{i}^{\text {out }}}\right)$ designates the variance of the set of grey-levels located on the external contour of the $I_{\mathrm{PA}}$ projection of the deformed template with a slightly inferior scale $k-\epsilon$ (and, respectively, slightly superior scale $k+\epsilon$ ) where $\epsilon \in[0,1]$ (e.g., Fig. 6). In our application, the log-likelihood energy term is expressed as

$$
E_{l}\left(s_{c}(\theta), I_{\mathrm{PA}}, I_{\mathrm{LAT}}\right)=E_{l}\left(s_{c}(\theta), I_{\mathrm{PA}}\right)+E_{l}\left(s_{c}(\theta), I_{\mathrm{LAT}}\right)
$$

where $E_{l}\left(s_{c}(\theta), I_{\mathrm{PA}}\right)$ and $E_{l}\left(s_{c}(\theta), I_{\mathrm{LAT}}\right)$ are log-likelihood energy terms for postero-anterior and lateral images respectively, with, for the postero-anterior image

$$
E_{l}\left(s_{c}(\theta), I_{\mathrm{PA}}\right)=\frac{1}{n_{\mathrm{PA}}} \sum_{\Gamma_{\mathrm{PA}}} \Psi_{\mathrm{PA}}(x, y)+\eta \sum_{i=1}^{n_{\mathrm{rib}}} \Upsilon_{i}
$$

In this expression:

- $\quad$ the first summation of $E_{l}\left(s_{c}(\theta), I_{\mathrm{PA}}\right)$ is over all the $n_{\mathrm{PA}}$ points of the external contour $\Gamma_{\mathrm{PA}}$ of the postero-anterior $\left(I_{\mathrm{PA}}\right)$ projection of the deformed template (e.g., Fig. 7) on a precomputed edge potential field estimated on $I_{\mathrm{PA}}$;

- $\quad$ the second summation is over all the $n_{\text {rib }}$ ribs of a rib cage;

- $\quad \eta$ is a factor allowing to control of the balance between the contour-based log-likelihood energy term and the regionbased log-likelihood energy term. 
This energy term is minimal when the external contour of the $I_{\mathrm{PA}}$ projection of each rib of the deformed template delineates two homogeneous regions (rib inside and rib outside) separated by a contour (e.g., Fig. 6).

On a lateral radiographic image $\left(I_{\mathrm{LAT}}\right)$, the ribs are hardly visible and difficult to identify. Moreover, the first rib is occluded by the second rib, and eleventh and twelfth ribs are almost completely hidden by the shadow of the abdomen. We cannot rely efficiently on the contours or the grey-level homogeneity of each rib as it has been done for $I_{\mathrm{PA}}$.

Instead, we use the log-likelihood term on $I_{\mathrm{LAT}}$ as a kind of constraint and we adopt the following strategy. We use the $K$-mean clustering procedure to segment $I_{\mathrm{LAT}}$ into two homogeneous regions, namely inside and outside the ribcage (with the class label $e_{\mathrm{in}}$ and $e_{\mathrm{out}}$ for the pixel belonging to the inside and, respectively, outside class). The log-likelihood energy term for lateral image is then defined by

$$
E_{l}\left(s_{c}(\theta), I_{\mathrm{LAT}}\right)=\frac{1}{n_{\mathrm{LAT}}} \sum_{s \in \Gamma_{\mathrm{LAT}}} \delta\left(e_{s}-e_{\mathrm{out}}\right)
$$

where $e_{s}$ designates the class label at site $s, e_{\text {out }}$ designates the class label for the pixel belonging to the outside rib cage class and the summation is overall the $n_{\text {LAT }}$ points of the external contour of the lateral projection $\left(s \in \Gamma_{\mathrm{LAT}}\right)$ of the deformed template on the segmentation of $I_{\mathrm{LAT}} . \delta(\cdot)$ is the delta dirac function for which $\delta(0)=1$ and $\delta(\neq 0)=0$.

This energy term is minimal when all the points of the external contour of the (laterally) projected deformed template belong to the "inside rib-cage" region.

\section{E. Three-Dimensional Reconstruction}

The only supervision required by our proposed reconstruction method consists in placing manually, using a simple graphical interface, a rectangular bounding box including the whole rib cage for the $I_{\mathrm{LAT}}$ and $I_{\mathrm{PA} 0}$ radiographic images. Using the center of the two rectangular bounding boxes, we estimate the 3 -D point coordinates corresponding to these two projected centers by the direct linear transformation (DLT) [5]. This estimated center is then considered as the center of the 3-D model. We then use the log-likelihood matching energy defined in Section IV-D in order to fit the two projections of an instance of the deformable model with the two corresponding radiographic images. (e.g., Fig. 7). This matching problem leads to a set of $K$ optimization problems (one for each detected class of pathological deformations) which is efficiently solved in our application with a stochastic optimization algorithm (see Section V). The optimal 3-D reconstruction corresponds to the solution, leading to the minimal energy, amongst these $K$ optimization problems, namely

$$
E_{\text {optimal }}\left(s_{c}(\theta)\right)=\arg \min _{c}\left\{\arg \min _{\theta} E\left(s_{c}(\theta)\right)\right\} .
$$

\section{OPTIMIZATION STRATEGY}

For each considered class-conditional prior model of pathological deformations $c(c \in[1, \ldots, K])$, the unsupervised 3-D reconstruction procedure is stated as an energy function minimization problem, namely

$$
\begin{aligned}
E\left(s_{c}(\theta)\right)=E_{l}\left(s_{c}(\theta), I_{\mathrm{PA}}\right)+E_{l}\left(s_{c}(\theta),\right. & \left.I_{\mathrm{LAT}}\right) \\
& +\beta E_{p}\left(s_{c}(\theta)\right)
\end{aligned}
$$

where $E_{l}$ is the log-likelihood energy term, $E_{p}$ is the prior energy term (or regularization term) restricting the deformation of the prior model $s_{c} . \beta$ is a factor allowing to control the balance between the two energy components and the rigidity of the deformable template, and $\theta$ are deformation parameters.

The energy function to be minimized is a complex function with several local extrema over the deformation parameter space. A global search is usually impossible due to the size of the configuration space. Instead, we have implemented the exploration/selection algorithm (E/S).

The E/S algorithm belongs to the class of evolutionary algorithms. This class of algorithm has been successfully applied in diverse areas such as medical imaging [35], localization of shapes [19], [23], nonphotorealistic rendering [36], and 3-D reconstruction of the scoliotic spine [9].

The E/S algorithm can be summarized as follows (more details are given in [37]). Let $F$ be a finite discrete subset of the Cartesian product of $\varsigma$ compact intervals $\left[m_{i}, M_{i}\right]$, for $1 \leq i \leq$ $\varsigma$, and $\theta=\left\{\theta_{1}, \ldots, \theta_{n}\right\}$ be a set of $n$ potential solutions randomly chosen. We define $\hat{\theta}$ as the minimal element $\theta_{i}$ of $\theta$ such that $E\left(s_{c}\left(\theta_{j}\right)\right)>E\left(s_{c}\left(\theta_{i}\right)\right)$, for $1 \leq j<i$, and $E\left(s_{c}\left(\theta_{j}\right)\right) \geq$ $E\left(s_{c}\left(\theta_{i}\right)\right)$, for $1<j \leq n$. We consider a graph $G$ defined on $\bar{F}$ called the exploration graph. The exploration graph is assumed to be nonoriented and connected. We denote by $\mathcal{N}(a)$ the neighborhood of the element $a \in F$ in the graph $G$ defined by [23] $\left\{b \in F\right.$ : for some $\left.j,\left|b_{j}-a_{j}\right| \leq r\left(M_{j}-m_{j}\right), b_{i}=a_{i}, i \neq j\right\}$ where $r$ is a real number in interval $[0,1]$ called the radius of exploration.

Each solution of $\theta$ is regarded as an individual that attempts a random search on the exploration graph [37]. For $b \in F$, we fix a positive distribution $a_{b}$ on $\mathcal{N}(b)$. The exploration process acts independently on each individual, and consists of choosing a random $N$ according to the binomial distribution $\operatorname{bin}(n, p)$ where $n$ is the size of the population and $p$ is the probability of exploration. We replace $\theta_{i}$ by $\vartheta_{i} \in \mathcal{N}\left(\theta_{i}\right) \backslash\{\hat{\theta}\}$ according to the uniform distribution, for $i \leq N$ otherwise, we change $\theta_{i}$ by $\hat{\theta}$. This process is running until a fixed maximum number of iterations has been reached.

\section{VALIDATION}

The validation of the accuracy of our 3-D reconstruction method is a difficult task because a scanned rib cage database is not available.

In our application, our 3-D reconstruction technique is validated by visual examination and also by comparing the reconstructed model obtained by our method and by the model resulting from the stereo-radiographic reconstruction technique developed in [6]. The validation procedure consists in fitting the model of our 3-D reconstruction method on the model resulting from the stereo-radiographic technique for the same scoliotic patient using quaternion based method of Horn [31]. 


\section{EXPERIMENTAL RESULTS}

\section{A. Rib Cages Database}

The training database consists of $532(u=532)$ scoliotic rib cages. All rib cages have the same number of ribs (11 right ribs and 11 left ribs). Midline of each rib is composed of 30 points. The 3-D coordinates of midline points of each rib cage were recorded in a specific order. The positions of the landmarks of all rib cages within the database are expressed in the same coordinate system.

The 3-D geometry of each rib cage within our database is obtained using the 3-D reconstruction technique developed in [6]. The accuracy of this technique was evaluated using radio-opaque markers. The mean and standard deviation were $1.01 \mathrm{~mm}$ and $0.72 \mathrm{~mm} \mathrm{[6].}$

\section{B. Radiographic Images}

In our application, we used two radiographic images (i.e., a postero-anterior with normal incidence and a lateral) acquired with a Fuji FCR 7501S radiographic imaging system. The radiographic image illustrates the superposition of the various structures on the same plan and gives place to semi-transparent images. The matrix size of our radiographic images is $2140 \times 880$ pixels (coded on 256 gray levels).

\section{Calibration}

In our application, the calibration of the radiological environment is done using the 3-D coordinates of each steel bead of calibration object previously measured and their corresponding 2-D observations on each radiographic views (position of each steel bead of calibration object preliminarily detected on each radiographic views) in order to solve the equation

$$
(x, y, \mu)^{T}=D(\varpi, \iota) \times(X, Y, Z)^{T}
$$

for each view. $\mu$ is the X-ray tube distance, $\varpi$ and $\iota$ being, respectively, the scale and rotation vectors. $(x, y)$ are the image coordinates of a point relative to the principal point. $(X, Y, Z)$ are the object space coordinates. Once the projection equations are calculated, the 3-D coordinates of any landmark identified and matched on more than one digital radiography are obtained by finding the intersection between each linear transformation of that particular point.

\section{Comparison Protocol}

We have validated our 3-D reconstruction method on a testing database (disjoint of the training database) of 20 pairs of calibrated radiographic images from scoliotic patients. We use the comparison protocol described in [38]. This comparison uses the distance (mean, and maximum) between a point from the reconstructed rib cage and the surface of the rib cage obtained with the stereo-radiographic reconstruction method [6].

\section{E. Experimental Results}

Fig. 2(a)-(c) shows reduced dimension of clusters as a function of the reconstruction error using different number of clusters when the rib cage training database is partitioned into $K=$ 8,10 , and 12 clusters respectively. In our application, we note
TABLE I

REDUCED DimENSION FOR EACH DETECTEd Class (Cluster) OF THE PPCA MIXTURE

\begin{tabular}{c|c}
\hline class & reduced dimension \\
\hline 1 & 19 \\
\hline 2 & 14 \\
\hline 3 & 11 \\
\hline 4 & 13 \\
\hline 5 & 10 \\
\hline 6 & 14 \\
\hline 7 & 14 \\
\hline 8 & 7 \\
\hline 9 & 16 \\
\hline 10 & 16 \\
\hline 11 & 16 \\
\hline 12 & 16 \\
\hline
\end{tabular}

that the criteria of component elimination presented in Section III-B does not allow to change the upper bound of the number of classes $K$ given to $K$-means for $\xi=0.01$. We also note that for a fixed $K$, the reduced dimension of each cluster decreases when the reconstruction error increases. The reduced dimensions, associated to each different class (for a reconstruction error ensuring less than $\varrho=17.5 \%$ error $^{4}$ ) is presented in Table I. Figs. 8 and 11 show two examples of 3-D reconstruction of rib cage of two scoliotic patients, for each detected class of pathological deformations. Figs. 9 and 12 show the optimal 3-D reconstruction corresponding to Figs. 8 and 11 respectively.

The 12 deformation classes of the rib cage learned on the training dataset are shown in Fig. 4. For the experiments, we have chosen $\beta=0.1$ for the weighting factor penalizing the prior energy term with respect to the log-likelihood energy term, and $\eta=1$ for the weighting factor allowing to control the balance between the two log-likelihood energies (contour-based and region-based).

We have used the Canny edge detector to estimate the edge map which is then used for the estimation of the edge potential fields on postero-anterior view (used in the log-likelihood energy term). In our application, $\sigma=1$, the mask size is $5 \times 5$, and the lower and upper thresholds are given by the unsupervised estimation technique proposed in [33]. For the E/S algorithm, we fix the size of population to 100 and the number of iterations to 100 .

The 3-D reconstruction of a scoliotic rib cage takes about 6 min on a $2.0-\mathrm{GHz}$ PC workstation running Linux. We have validated our 3-D reconstruction method on a database of 20 pairs of calibrated radiographic images (postero-anterior and lateral) of scoliotic patients. The mean, and maximum errors between points from the reconstructed rib cage and the surface of the rib cage obtained with stereo-radiographic are, respectively, $(1.62 \pm 0.50) \mathrm{mm}$, and $(3.62 \pm 1.02) \mathrm{mm}$.

\footnotetext{
${ }^{4}$ Let us clarify that it is not a geometrical reconstruction error. If $V_{T}$ is the sum of the eigenvalues, then the number $m$ of eigenvalues to be selected is such that
}

$$
\frac{\sum_{i=1}^{m} \lambda_{i}}{V_{T}} \geq(1-\varrho) .
$$


class $1(E=-0.3482)$

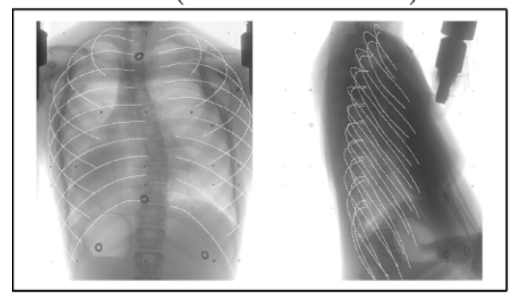

class $3(E=-0.3815)$

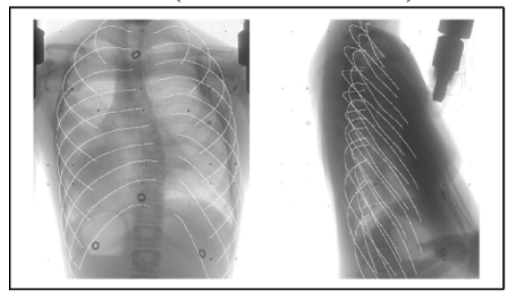

class $5(E=-0.5568)$

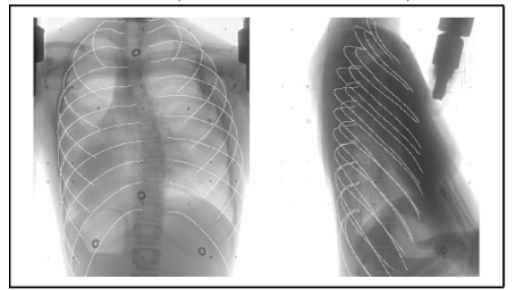

class $7(E=-0.7200)$

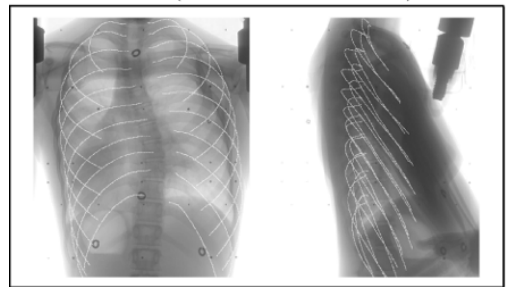

class $9(E=-0.5523)$

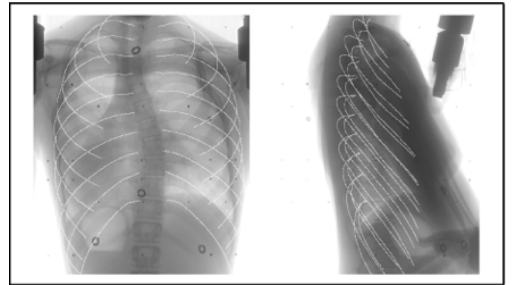

class $11(E=-0.4551)$

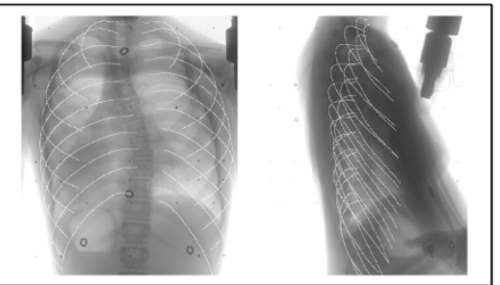

class $2(E=-0.4523)$

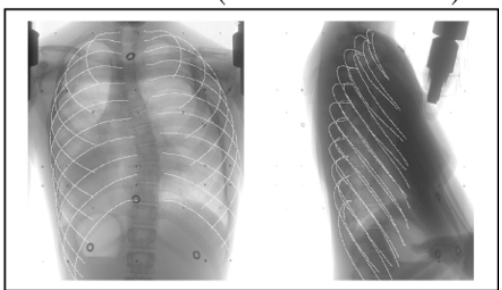

class $4(E=-0.8098)$

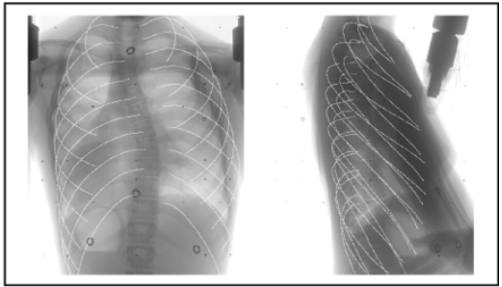

class $6(E=-0.8179)$

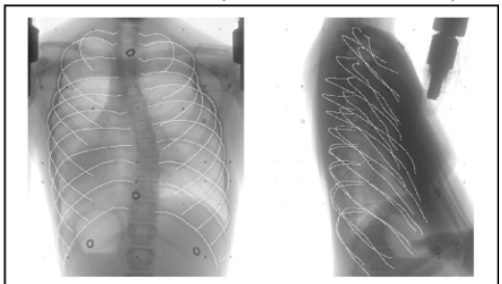

class $8(E=-0.5568)$

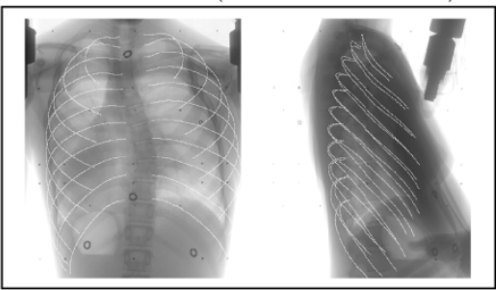

class $10(E=-0.7560)$

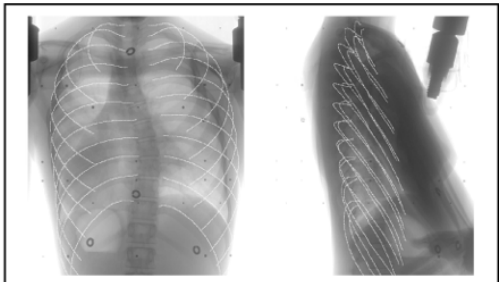

class $12(E=-0.4901)$

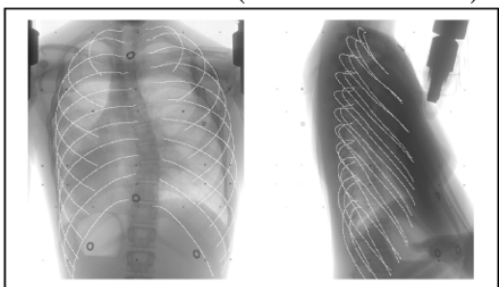

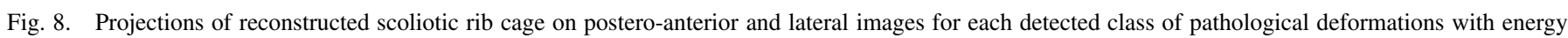
value corresponding. The optimal 3-D reconstruction corresponds to the class 6 .

\section{DISCUSSION AND CONCLUSION}

In this paper, we have presented a new technique for the 3-D reconstruction of scoliotic rib cage from two radiographic projections $I_{\mathrm{PA}}$ and $I_{\mathrm{LAT}}$. This method efficiently exploits the es-timation of a mixture of PPCA for both dimensionality reduction and to constrain the 3-D reconstruction problem. The proposed 3-D reconstruction problem is viewed as a set of optimization problems, each one associated and constrained by a specific class of pathological deformations observed on a representative training scoliotic rib cage population and efficiently detected by the MPPCA method. Our 3-D reconstruction 


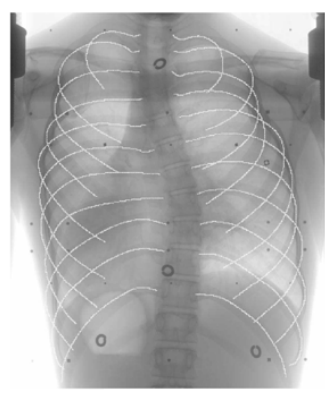

(a)

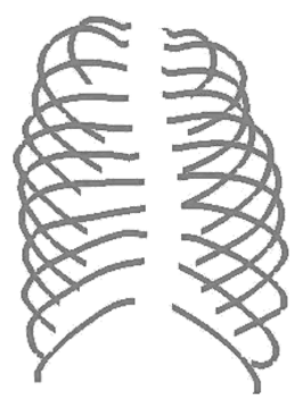

(c)

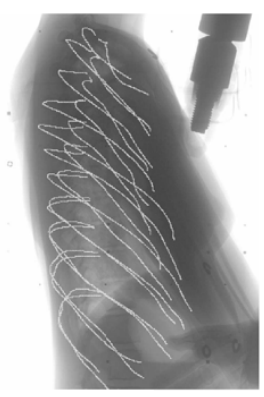

(b)

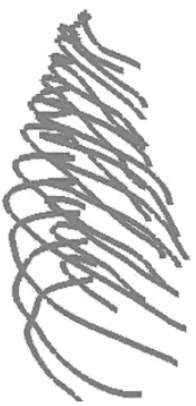

(d)
Fig. 9. Optimal 3-D reconstruction corresponds to the class 6 in Fig. 8 . (a) Projections of reconstructed scoliotic rib cage on postero-anterior image. (b) Projections of reconstructed scoliotic rib cage on lateral image. (c) and (d) Visualization of the reconstructed scoliotic rib cage from the coronal and sagital view.

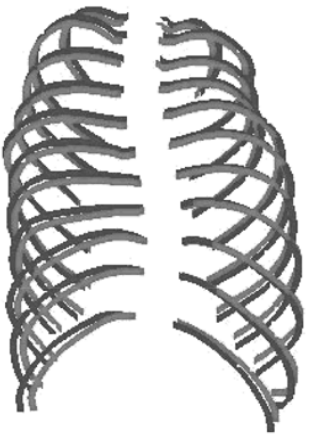

(a)

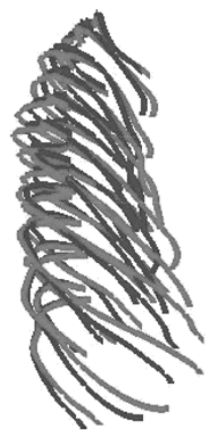

(b)
Fig. 10. Visual comparison between the 3-D reconstruction using our method and reference stereo-radiographic corresponds to the class 6 in Fig. 8 . (a) Visualization of the two reconstructed rib cages from the coronal view. (b) Visualization of the two reconstructed rib cages from the sagital view.

method has the advantage of minimizing the X-ray dose (only two radiographic images) and the time needed for digitization and supervision required when compared to that of other reconstruction methods. The results obtained in our experiments are quite encouraging and indicate that accurate unsupervised 3-D reconstruction is technically feasible. This is an important improvement compared to the method presented by Mouren [10] or [6].

Let us note that there are several classification systems of spinal deformities [39], but these systems could not be applied for the classification of the rib cage deformities. In our application, the number $K$ of deformation classes (i.e., the number of components in the mixture of PPCA) is chosen empirically, namely $K=12$, by inspection of the rib cage database and also by the good reconstruction results (in term of accuracy) that we are able to obtain. An extension of this work would be to automatically estimate the number of class of the deformation mixture by using information theoretic criteria (AIC or MDL) [17], [18], [25], [26].

This method has been validated on a sample of 7 isolated ribs, by comparing the model obtained from stereo-radiographic method and those obtained with radio-opaque markers. The mean and standard deviation were 1.01 and $0.72 \mathrm{~mm}$. It has also been validated on a sample of 24 ribs by two digitalizations of the different costal lines. The mean difference between the two 3-D models obtained was $1.22 \mathrm{~mm}$ and standard deviation was $1.04 \mathrm{~mm}$. In our validation, we have used this supervised 3-D reconstruction method (whose procedure is summarized in the introduction) as reference for the validation of our reconstruction model. Figs. 10 and 13 show visual comparison between 3-D reconstruction using our method and reference stereoradiographic. The mean, and maximum errors of our method are, respectively, $(1.62 \pm 0.50) \mathrm{mm}$, and $(3.62 \pm 1.02) \mathrm{mm}$.

Compared to our 3-D reconstruction technique, the biplanar technique proposed in [10] is significantly more supervised and operator-dependent; it requires to manually identify and digitize a set of 60 points in the lateral view. Moreover, the PCA used in [10] only defines a linear dimensionality reduction which is a strong and not necessarily a true assumption in this context. This method has been only validated on a sample of 20 pairs of synthetic images (in which the authors suppose that poissonian noise in the image is stationary and parameters are known). In their case, the mean and maximum errors are respectively $(2.0 \pm$ $0.3) \mathrm{mm}$, and $(18.5 \pm 2.3) \mathrm{mm}$.

Let us recall that the stereo-radiographic technique presented in [6] is also limited due to the inherent inaccuracy produced in identifying 11 points per rib on $I_{\mathrm{PA}}$ and $I_{\mathrm{PA}-20^{\circ}}$ (leading to reconstruction errors). Locating these features is widely supervised and, therefore, time-consuming (up to two hours). Moreover, this method does not exploit all information contained in the two X-ray radiographic projections, for instance, the contours of each rib, the geometrical structure or the statistical knowledge of the possible deformation of the rib cage to be reconstructed.

By comparison, our method answers the above-mentioned limitations of these techniques. All the experiments are carried out with the same parameters which are determined empirically but are reliable and robust for all the tested experiments. For example, the number $K$ of deformation classes (i.e., the number of components in MPPCA) is learned by the inspection of the rib cage database and by using the classification process of the scoliosis pathology used at Hospital Sainte-Justine's. ${ }^{5}$ The weighting factor $\beta$ for penalizing the prior energy term with respect to the log-likelihood energy term and the weighting factor $\eta$ for controlling the balance between two log-likelihood energies (contour-based and region-based) are determined empirically and are valid for this imaging modalitie. Finally, the

\footnotetext{
53D Scoliosis Computing Laboratory (LIS-3D), Sainte-Justine Mother-Child University Hospital, Montréal, QC, Canada.
} 
class $1(E=-0.4560)$

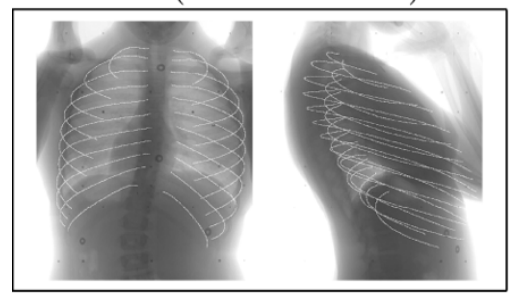

class $3(E=-0.3627)$

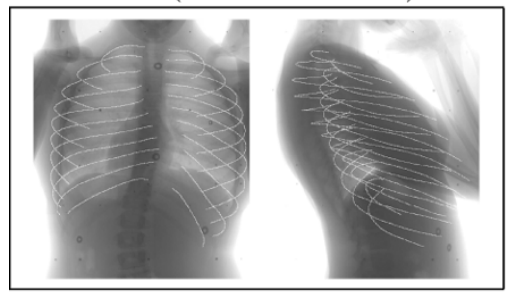

class $5(E=-0.4702)$

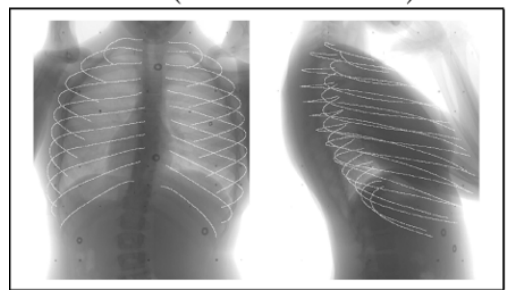

class $7(E=-0.3568)$

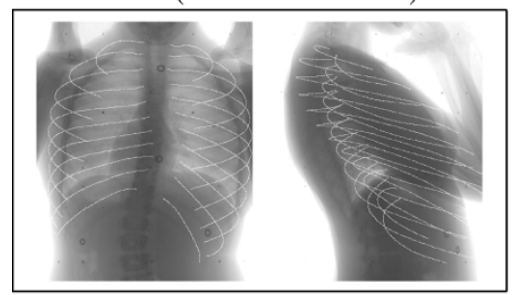

class $9(E=-0.8754)$

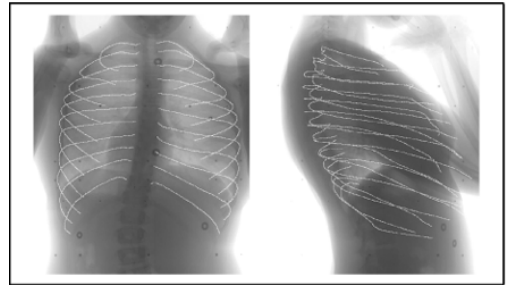

class $11(E=-0.6118)$

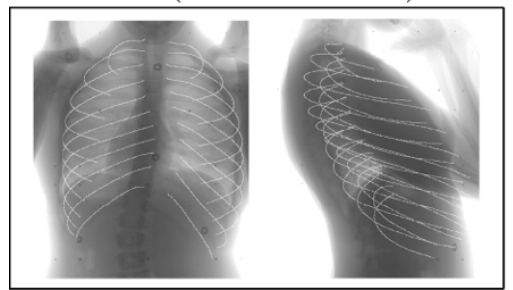

class $2(E=-0.7118)$

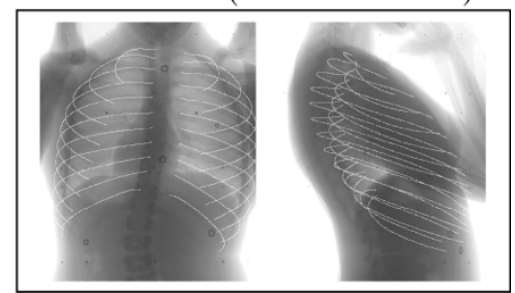

class $4(E=-0.7052)$

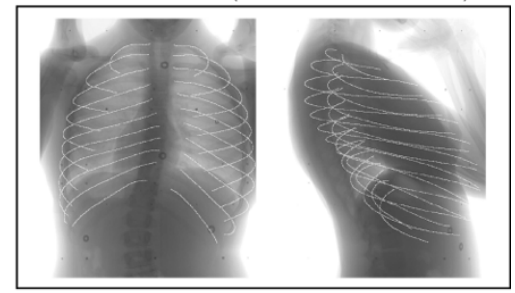

class $6(E=-0.6006)$

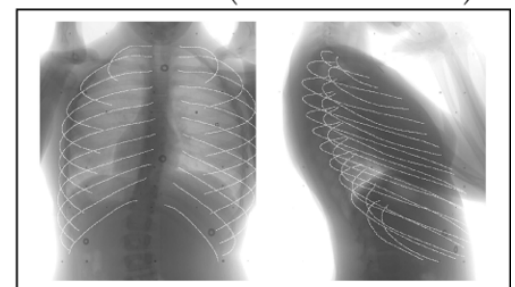

class $8(E=-0.4683)$

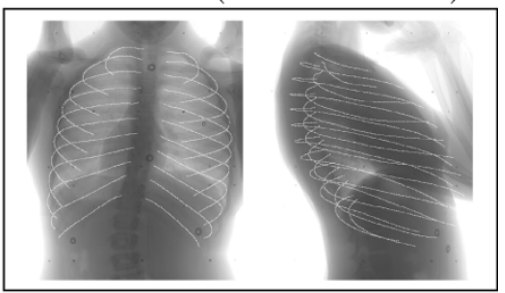

class $10(E=-0.5423)$

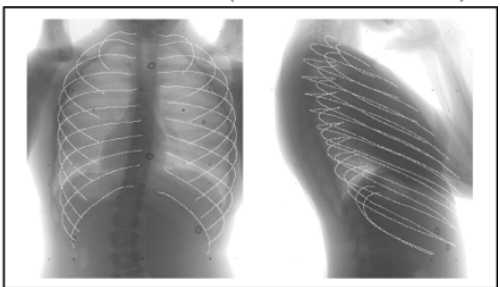

class $12(E=-0.6584)$

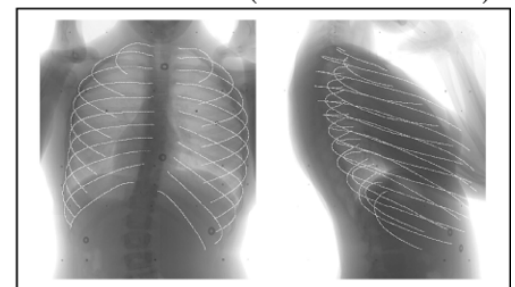

Fig. 11. Projections of reconstructed scoliotic rib cage on postero-anterior and lateral images for each detected class of pathological deformations with the corresponding energy value. The optimal 3-D reconstruction corresponds to the class 9 .

proposed method requires a training representative database. Nevertheless, the proposed reconstruction technique remains unsupervised in the sense that this database is constructed off-line and not during the $3-\mathrm{D}$ reconstruction step.

Our method offers an accurate representation of the rib cage from only two radiographic views, whereas the computed to- mography (CT)-scan requires between 200 and 400 images to obtain the same 3-D reconstruction with the same accuracy. The proposed method is thus interesting as for the quantity of data to be acquired, processed and managed. For example, two digital Fuji X-Rays require 15 Mbytes $(1760 \times 2140 \times 2$ bytes $)$ of storage in comparison to 200 Mbytes $(512 \times 512 \times 2$ bytes 


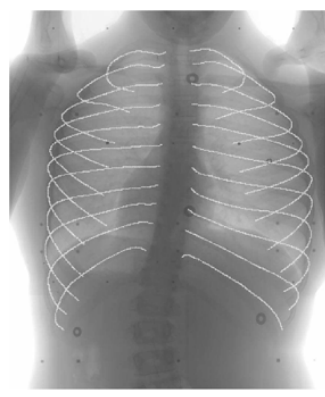

(a)

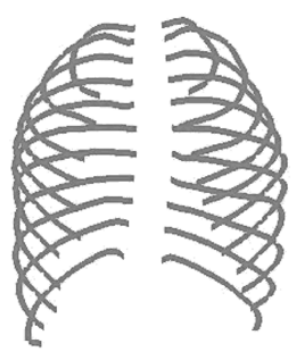

(c)

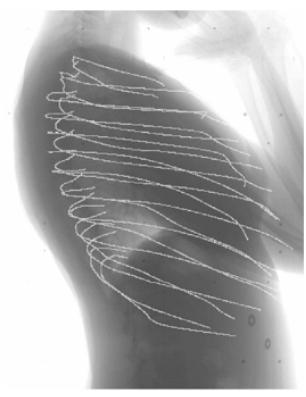

(b)

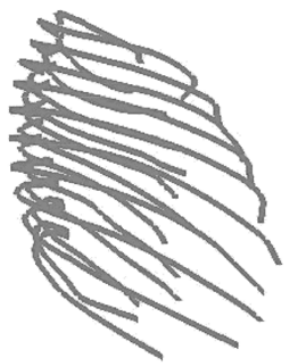

(d)
Fig. 12. Optimal 3-D reconstruction corresponding to the class 9 in Fig. 11. (a) Projections of reconstructed scoliotic rib cage on postero-anterior image. (b) Projections of reconstructed scoliotic rib cage on lateral image. (c) and (d) Visualization of the reconstructed scoliotic rib cage from the coronal and sagital view.

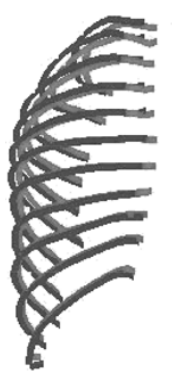

(a)

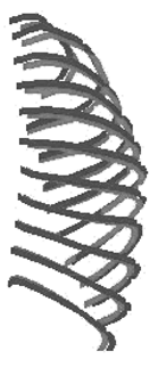

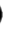

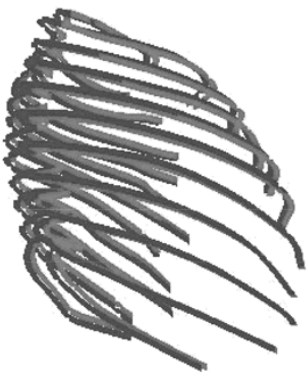

(b)
Fig. 13. Visual comparison between 3-D reconstruction using our method and reference stereo-radiographic (corresponding to the class 9 in Fig. 11). (a) Visualization of the two reconstructed rib cages from the coronal view. (b) Visualization of the two reconstructed rib cages from the sagital view.

$\times 400$ slices) for a CT-scan reconstruction technique. The effective dose ${ }^{6}$ in chest $\mathrm{CT}$ is about 8 milliseiverts $(\mathrm{mSv})$, around 400 times more than chest digital $\mathrm{X}$-rays dose (chest radiographic examination: $0.02 \mathrm{mSv}$ ) [40]. The absorbed dose with CT can often approach or exceed the levels known to increase the probability of cancer as shown in epidemiological studies [40],). With the generalization of multi-detectors spiral CT, ${ }^{7}$ patients will be

\footnotetext{
${ }^{6}$ Sum of the products of the dose equivalent to the organ or tissue and the weighting factor for each organ or tissue irradiated [40].

${ }^{7}$ Radiation dose in multislice cone-beam helical CT depends upon the choice of several factors. Even though it is possible to perform a helical CT with lower radiation dose than slice-by-slice CT, in practice the patient gets higher dose due to the factors chosen (scan volume, mAs, pitch, slice width). An increase of radiation dose by 10\%-30\% may occur with multi-slice detector arrays for multi-slice CT [40].
}

more and more exposed to radiations and a method that can contribute to keep as lower as possible this amount of radiation is also quite important and should be considered. This becomes especially important when we deal with young scoliotic patients that will be exposed to multiple diagnostic radiographic examinations during childhood and adolescence [41].

Our proposed scheme thus constitutes an alternative to CT-scan 3-D reconstruction with the advantage of low irradiation and will be of great interest for diagnosis of spinal deformities, simulation of orthopedic treatments, and for reliable geometric models for finite element studies. However, this reconstruction method is not suitable without improvement for surgical navigation applications when compared to CT-scan reconstruction errors of $\pm 1 \mathrm{~mm}$, the gold standard for those applications.

The proposed method remains sufficiently general to be applied to other medical reconstruction problems (i.e., pelvis, knee, etc.) for which a database of the anatomical structure is available (with two or more radiographic views).

\section{ACKNOWLEDGMENT}

The authors would like to thank the anonymous reviewers for helpful comments and criticisms which have greatly improved the paper. The authors thank the Natural Sciences and Engineering Research Council of Canada, Canadian Foundation for Innovation, Valorisation recherche Québec and the Biospace Company, Paris, France, for supporting this study. The authors would also like to thank H. Labelle, full professor at the surgery department of the University of Montréal and pediatric orthopaedic surgeon, for having provided the rib cages database and for helpful discussions, and L. Normandeau medical physician, responsible for radiological protection at the Hospital Center of the University of Montréal (CHUM) for having provided the references of patient radiation dose for CT-scan and conventional radiography-based imaging modalities.

\section{REFERENCES}

[1] S. Delorme, P. Violas, J. Dansereau, J. de Guise, C. Aubin, and H. Labelle, "Preoperative and early postoperative three-dimensional changes of the rib cage after posterior instrumentation in adolescent idiopathic scoliosis," Eur. Spine J., vol. 10, pp. 101-106, 2000.

[2] B. Carvalho and G. Herman, "Helical CT reconstruction from wide cone-beam angle data using ART," in Proc. XVI Brazilian Symp. Computer Graphics and Image Processing, São Carlos, São Paulo, Brazil, Oct. 2003, pp. 363-370.

[3] A. Reeves, D. Yankelevitz, and C. Henschke, "Three-dimensional segmentation and growth-rate estimation of small pulmonary nodules in helical CT images," IEEE Trans. Med. Imag., vol. 22, no. 10, pp. 1259-1274, Oct. 2003.

[4] J. Lötjönen, P.-J. Reissman, I. Magnin, and T. Katila, "Model extraction from magnetic resonance volume data using the deformable pyramid," Med. Image Anal., vol. 3, no. 4, pp. 387-406, 1999.

[5] S. Sandor and R. Leahy, "Surface-based labeling of cortical anatomy using a deformable atlas," IEEE Trans. Med. Imag., vol. 16, no. 1, pp. 41-54, Feb. 1997.

[6] J. Dansereau and I. Stokes, "Measurements of three dimensional shape of the rib cage," J. Biomech., vol. 21, pp. 893-901, 1988.

[7] G. Marzan, "Rational Design for Close-Range Photogrammetry," Ph.D. dissertation, Dept. Civil Eng., Univ. Illinois at Urbana-Champaign, Urbana, 1976 . 
[8] E. Marcil, J. Dansereau, H. Labelle, and J. D. Guise, "Incorporation of patient displacement into a trunk reconstruction technique," in Proc. IEEE 17th Annu. Conf. Engineering in Medicine and Biology Society, vol. 1, Sep. 1995, pp. 385-386.

[9] S. Benameur, M. Mignotte, S. Parent, H. Labelle, W. Skalli, and J. D. Guise, "A hierarchical statistical modeling approach for the unsupervised 3-D reconstruction of the scoliotic spine," in Proc. 10th IEEE Int. Conf. Image Processing, vol. 1, Barcelona, Spain, Sep. 2003, pp. $561-564$.

[10] S. Mouren, "Reconstruction 3-D Biplanaire de Cages Thoraciques Scoliotiques à l'aide de modèles déformables de côtes," Master's thesis, Dept. Mech. Eng., École Polytechnique, Montréal, QC, Canada, 2003.

[11] M. Tipping and C. Bishop, "Mixtures of probabilistic principal component analyzers," Neural Computation, vol. 11, no. 2, pp. 443-482, 1999.

[12] G. Celeux and J. Diebolt, "The SEM algorithm: A probabilistic teacher algorithm derived from the EM algorithm for the mixture problem," Computational Statist. Quart., vol. 2, pp. 73-82, 1985.

[13] J. Diebolt and G. Celeux, "Asymptotic properties of a stochastic EM algorithm for estimating mixture proportions," Stochastic Models, vol. 9, pp. 599-613, 1993.

[14] B. Delyon, M. Lavielle, and E. Moulines, "Convergence of a stochastic approximation version of the EM algorithm," Ann. Statist., vol. 27, no. 1, pp. 94-128, 1999.

[15] D. Chauveau, "A stochastic EM algorithm for mixtures with censored data," J. Statistical Planning Inference, vol. 46, pp. 1-25, 1995.

[16] G. Celeux, D. Chauveau, and J. Diebolt, "Stochastic versions of the EM algorithm," J. Statistical Computation Simulat., vol. 55, pp. 287-314, 1996.

[17] T. Lei and W. Sewchand, "Statistical approach to X-ray CT imaging and its applications in image analysis-II: A new stochastic model-based image segmentation technique for X-ray CT image," IEEE Trans. Med. Imag., vol. 11, no. 1, pp. 62-69, Mar. 1992.

[18] T. Lei and J. Udupa, "Performance evaluation of finite normal mixture model-based image segmentation techniques," IEEE Trans. Image Process., vol. 12, no. 10, pp. 1153-1169, Oct. 2003.

[19] F. Destrempes and M. Mignotte, "Unsupervised localization of shapes using statistical models," in Proc. Int. Conf. Signal and Image Processing, Kauai, HI, Aug. 2002, pp. 60-65.

[20] C. Drexler, F. Mattern, and J. Denzler, "Hierarchic object models and classification based on probabilistic PCA," in Proc. IAPR Workshop Machine Vision Applications, Nara, Japan, Dec. 2002, pp. 435-438.

[21] K. Grauman, G. Shakhnarovich, and T. Darrell, "Inferring 3-D structure with a statistical image-based shape model," in Proc. IEEE Int. Conf. Computer Vision, Nice, France, Oct. 2003, pp. 641-648.

[22] M. Tipping and C. Bishop, "Probabilistic principal component analysis," J. Roy. Statist. Soc., ser. B, vol. 21, no. 3, pp. 611-622, 1999.

[23] F. Destrempes, "Détection non-supervisée de contours et localization de formes à l'aide de modèles statistiques," Master's thesis, Dept. Comput. Sci. Operations Res., University of Montréal, Montréal, QC, Canada, 2002.

[24] J. Hartigan and M. Wong, "A $K$-means clustering algorithm," Appl. Statist., vol. 28, pp. 100-108, 1979.

[25] G. Celeux and G. Soromenho, "An entropy criterion for assessing the number of clusters in a mixture model," J. Classification, vol. 13, pp. $195-212,1996$

[26] M. Miloslavsky and M. van de Laan, "Fitting of mixtures with unspecified number of components using cross validation distance estimate," Computational Statist. Data Anal., vol. 41, pp. 413-428, 2003.

[27] O. Zamir and O. Etzioni, "Web document clustering: A feasibility demonstration," in Proc. 21st Annu. Int. ACM SIGIR Conf. Research and Development in Information Retrieval, Melbourne, Australia, Aug. 1998, pp. 46-54.

[28] S. Geman and D. Geman, "Stochastic relaxation, Gibbs distributions and the Bayesian restoration of images," IEEE Trans. Pattern Anal. Mach. Intell., vol. PAMI-6, pp. 721-41, 1984.

[29] G. Golub and C. V. Loan, Matrix Computations, 3rd ed. Baltimore, MD: Johns Hopkins Univ. Press, 1996.

[30] T. Cootes and C. J. Taylor, "A mixture model for representing shape variation," in Proc. Br. Machine Vision Conf., vol. 1, Essex, U.K., 1997, pp. 110-119.

[31] B. Horn, H. Hilden, and S. Negahdaripour, "Closed-form solution of absolute orientation using orthonormal matrices," J. Opt. Soc. Am. A, vol. 5, no. 7, pp. 1127-1135, 1988.
[32] P. Besl and N. McKay, "A method for registration of 3-D shapes," IEEE Trans. Pattern Anal. Mach. Intell., vol. 14, no. 2, pp. 239-256, Feb. 1992.

[33] J. Canny, "A computational approach to edge detection," IEEE Trans. Pattern Anal. Mach. Intell., vol. PAMI-8, no. 6, pp. 679-697, Jun. 1986.

[34] A. Jain, Y. Zhong, and S. Lakshmanan, "Object matching using deformable templates," IEEE Trans. Pattern Anal. Mach. Intell., vol. 18, no. 3, pp. 267-278, Mar. 1996.

[35] M. Mignotte, J. Meunier, and J.-C. Tardif, "Endocardial boundary estimation and tracking in echocardiographic images using deformable templates and Markov random fields," Pattern Anal. Applicat., vol. 4, no. 4, pp. 256-271, 2001.

[36] M. Mignotte, "Unsupervised statistical sketching for nonphotorealistic rendering models," in Proc. 10th IEEE Int. Conf. Image Processing, vol. 3, Barcelona, Spain, Sep. 2003, pp. 573-577.

[37] O. Fancois, "Global optimization with exploration/selection algorithms and simulated annealing," Ann Appl Probability, vol. 12, pp. 248-271, 2002.

[38] S. Benameur, M. Mignotte, S. Parent, H. Labelle, W. Skalli, and J. D. Guise, "3D/2D registration and segmentation of scoliotic vertebrae using statistical models," Computerized Med Imag Graphics, vol. 27, no. 5, pp. 321-337, 2003.

[39] G. Wiggins, C. Shaffrey, M. Abel, and A. Menezes, "Pediatric spinal deformities," J Neurosurg Am Assoc Neurological Surgeons, vol. 14, no. 1, pp. 1-14, 2003.

[40] M. Rehani, G. Bongartz, W. Kalender, S. Golding, L. Gordon, T. Murakami, and P. Shrimpton, "Managing Patient Dose in Computed Tomography," Annals of the International Commission on Radiological Protection, Int. Comm. Radiological Protection (ICRP) 87, vol. 30, 2001.

[41] M. Doody, J. Lonstein, M. Stovall, D. Hacker, N. Luckyanov, and C. Land, "Breast cancer mortality after diagnostic radiography: Findings from the U.S. scoliosis cohort study," Spine, vol. 25, no. 16, pp. 2052-2063, 2000.

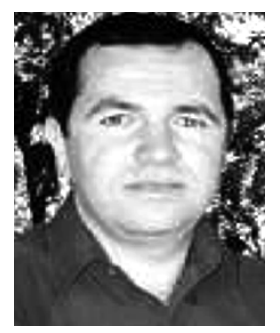

Said Benameur was born in Tizi-Ouzou, Algeria. He received the Diploma Engineer degree in computer engineering from the Mouloud Mammeri University, Tizi-Ouzou, Algeria, in 1990, the M.Sc. degree in computer engineering from University of Quebec, Montréal (UQAM), QC, Canada, in 1998, and the Ph.D. degree in engineering from Engineering School (ETS), Quebec University, Canada, in 2004.

From 1993 to 1996, he was Computer engineer at the Head quarters civil, Algeria; He is currently a Postdoctoral Fellow in the Computer Science and Operations Research department of the University of Montréal working at the Image Processing Laboratory. His current research interests include statistical methods, deconvolution and restoration, and computer vision and its applications to medical imaging.

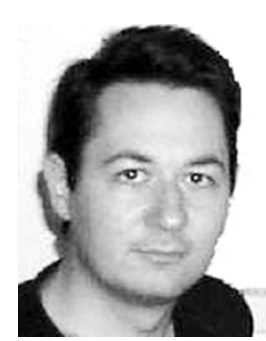

Max Mignotte received the Master of Sciences degree in electronics and telecommunications in 1992 and the DEA postgraduate degree in digital signal, image, and speech processing from the INPG University, Grenoble, France, in 1993 and the Ph.D. degree in electronics and computer engineering from the University of Bretagne Occidentale (UBO) and the digital signal laboratory (GTS) of the French Naval academy, France, in 1998.

He was an INRIA postdoctoral fellow at the University of Montréal, Montréal, QC, Canada (DIRO), Canada, from 1998 to 1999. he was also a NSERC Postdoctoral Fellow, a Teaching Assistant and a Lecturer at the University of Montréal from September 1999 to July 2000. He is currently with DIRO at Computer Vision and Geometric Modeling Lab as an Assistant Professor at University of Montréal. His current research interests include statistical methods and Bayesian inference for image segmentation (with hierarchical Markovian, statistical templates, or active contour models), parameters estimation, tracking, classification, deconvolution, and restoration issues in medical or sonar imagery. 


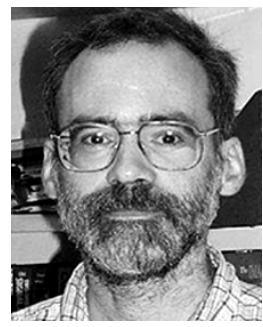

François Destrempes received the B.Sc. degree in mathematics from the Université de Montréal, Montréal, QC, Canada, in 1985. He received the M.Sc. and the Ph.D. degrees in mathematics from Cornell University, Ithaca, NY, in 1990. He also received a postgraduate degree (2000) in applied computer science and the M.Sc. degree (2002) in computer science from the Université de Montréal, where he is now studying in the Ph.D. program in computer science.

He was a Postdoctoral Fellow with the Centre de Recherche Mathématiques (CRM), Université de Montréal, Montréal, QC, Canada, from 1990-1992. He has taught mathematics at Concordia University, the University of Ottawa, the University of Toronto, and the University of Alberta. His current research interests include statistical methods for image segmentation, parameters estimation, detection of contours, localization of shapes, and applications of stochastic optimization to computer vision.

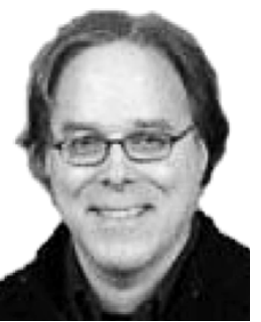

Jacques A. De Guise received the B.Sc. degree in electrical engineering and the Ph.D. degree in biomedical engineering from École Polytechnique of Montréal, Montréal, QC, Canada, in 1977 and 1984, respectively.

He was a Natural Sciences and Engineering Research Council (NSERC) Postdoctoral Scholar at the Computer Vision and Robotics Laboratory of McGill University (Canada) from 1984 to 1986 . He was a NSERC Researcher Fellow at the Institut de génie biomédical of the University of Montréal from 1986 to 1990 . He is currently Full Professor at the Automated Production Department of the École de technologie supérieure of Montréal and Director of the Laboratoire de recherche en imagerie et orthopédie (LIO) of the University of Montréal Hospital Research Centre. He is Chair holder of the Canada Research Chair in 3-D imaging and biomedical engineering. His current research interests are 3-D medical imaging, 3-D modeling of the musculoskeletal and vascular systems, and computer assisted surgery. 